TRANSACTIONS OF THE

AMERICAN MATHEMATICAL SOCIETY

Volume 353, Number 3, Pages 1183-1219

S 0002-9947(00)02582-4

Article electronically published on November 8, 2000

\title{
BEYOND BORCHERDS LIE ALGEBRAS AND INSIDE
}

\author{
STEPHEN BERMAN, ELIZABETH JURISICH, AND SHAOBIN TAN \\ This paper is dedicated to Professor Peter Slodowy
}

\begin{abstract}
We give a definition for a new class of Lie algebras by generators and relations which simultaneously generalize the Borcherds Lie algebras and the Slodowy G.I.M. Lie algebras. After proving these algebras are always subalgebras of Borcherds Lie algebras, as well as some other basic properties, we give a vertex operator representation for a factor of them. We need to develop a highly non-trivial generalization of the square length two cut off theorem of Goddard and Olive to do this.
\end{abstract}

\section{INTRODUCTION}

In this paper we define and study a new class of Lie algebras, given by generators and relations, which simultaneously generalize the Borcherds Lie algebras and the generalized intersection matrix algebras of Slodowy. Throughout the paper we will refer to the generalized intersection matrix algebras simply as G.I.M. algebras. Both of these classes arise by attaching a Lie algebra to a matrix, and both generalize the notion of a Kac-Moody Lie algebra but from different perspectives. In the case of a Borcherds Lie algebra one begins with a real symmetrizable matrix, the major difference between these and the Kac-Moody algebras is that imaginary roots, as opposed to just real roots, are allowed to be simple roots. In the case of Slodowy G.I.M. algebras one begins with an integral matrix with twos on the major diagonal, so all simple roots are real as in the Kac-Moody case, but here one allows the off diagonal entries of the matrix to be positive and then adjusts the relations corresponding to these entries. The Borcherds algebras have a theory (see [Bo1], [Bo2], [Bo3], [J1], [J2], [JLW], [K]) which closely resembles Kac-Moody theory, and in particular the algebras have a triangular decomposition in the sense of [MP] which reflects the fact that the roots are either positive, negative, or zero. Slodowy algebras on the other hand, do not have any natural triangular decomposition since the roots, when expressed as a linear combination of the simple roots, may have both positive and negative coefficients. Our theory here will present a common framework for both of these cases. However, the reader should note that since Borcherds algebras are quite like Kac-Moody algebras, and since Slodowy G.I.M. algebras do not necessarily resemble Kac-Moody algebras, our viewpoint here is to present a "G.I.M. version" of Borcherds algebras. Thus, our results follow more

Received by the editors March 18, 1998 and, in revised form, May 7, 1999.

2000 Mathematics Subject Classification. Primary 17B65; Secondary 17B69.

The first auther gratefully acknowledges the support of the Natural Sciences and Engineering Research Council of Canada. 
closely those found in the theory of Slodowy G.I.M. algebras than the theory of Borcherds algebras, although they of course apply to both cases.

Recall that in the theory of G.I.M. algebras one may reduce to the case of algebras attached to indecomposable matrices, and then one shows that such an algebra is isomorphic to a subalgebra of a Kac-Moody algebra consisting of fixed points of a period two automorphism. In fact, there are two cases here, the oriented case, dealt with in [S11], [S12] and the unoriented case dealt with in [Be. Thus, a G.I.M. algebra is inside a Kac-Moody Lie algebra which is called the double of the original algebra. In the oriented case the Kac-Moody algebra splits into a direct sum of two isomorphic smaller Kac-Moody algebras. Call this smaller Kac-Moody algebra $\mathcal{L}$. One then finds the original G.I.M. algebra is also isomorphic to $\mathcal{L}$. Thus, in the oriented case the G.I.M. algebra is a Kac-Moody algebra. In the unoriented case the Kac-Moody algebra has its Cartan matrix indecomposable and one knows that, at least in some sense, the G.I.M. algebra is far from being graded simple. This prompted Slodowy to consider a factor algebra of the G.I.M. algebra in the case when the original matrix defining the algebra is symmetric. Thus, he let $\mathcal{R}$ be the ideal of the G.I.M. algebra generated by all root spaces, $\mathcal{G}_{\alpha}$, where the root $\alpha$ satisfies $(\alpha, \alpha)>2$, and the corresponding factor algebra is then called an I.M. algebra. It is very interesting that there are some quite non-trivial realizations known for certain of these I.M. algebras (see $[\overline{B M}]$, [BZ]), but in general very little is known about their structure. In fact, from the very definition, since it is given by factoring by $\mathcal{R}$, it is not at all clear whether or not the I.M. algebra is nonzero. This problem was solved in the interesting paper [EMY] where the authors provided a non-trivial vertex operator representation for an I.M. algebra hence showing such an algebra is non-zero. One of the key results they used in doing this is the so-called square length two cut off theorem of Goddard and Olive [GO], and the authors remarked on how perfectly this result fits with the definition of $\mathcal{R}$ given by Slodowy in defining the I.M. algebras.

Our purpose then, in this paper, is to bring the algebras which we study to this same degree of development. We begin in Section 2 by defining what we call an admissible matrix $A$ and then show how to attach a Lie algebra, $\mathcal{G}(A)$, to it. Some care must be taken in making the basic definition of $\mathcal{G}(A)$ via generators and relations. After that the basic properties which we establish follow almost exactly as in the G.I.M. algebra case and so we are brief, and often content ourselves with giving references to the basic works there ([Sl1], [S12], [Be], [EMY]). Following Slodowy's work in the G.I.M. case we show how some of the algebras $\mathcal{G}(A)$ can be related to the notion of affinizing a Lie algebra and we close the section by defining the ideal $\mathcal{R}$ and its associated factor algebra, $\mathcal{L}(A)$, when the admissible matrix $A$ is symmetric and has even integer entries on its main diagonal.

In the third and final section we construct a module $M$ for $\mathcal{G}(A)$ which satisfies $\mathcal{R} M=(0)$ in the case when $A$ is symmetric and has even integers on the diagonal and thus, we obtain a representation of $\mathcal{L}(A)$. This generalizes the work in [EMY] but it is a highly non-trivial generalization for the following reasons. First, the vertex operators, $X(\alpha, z)$, used in [EMY] satisfy the well-known identity $[X(\alpha, z), X(-\alpha, w)]=0$ if $(\alpha, \alpha) \leq 0$ (see [FLM] $)$ and hence they cannot be associated to any imaginary simple roots $\alpha$ we might have as generators of $\mathcal{G}(A)$ which satisfy this condition. That is, we need to find a new type of operator to associate to simple roots $\alpha$ which are not real roots. Moreover, we must do this in such a way that the Goddard-Olive result remains true in our setting. A hint 
at how to make the basic definitions comes from [Fr, Proposition 2.3] and [FLM], 8.5.5] where normally ordered vertex operators of the form $\beta(z) X(\alpha, z)$ are considered. We let $X^{(\beta)}(\alpha, z)$ denote this operator. In our case, we let $\left\{\alpha_{i}\right\}_{i \in I}$ denote all simple roots of $\mathcal{G}(A)$ and then let $\left\{\alpha_{j}\right\}_{j \in J}$ denote the subset of simple roots which are not real (so satisfy $\left(\alpha_{j}, \alpha_{j}\right) \leq 0$ ). We then form two lattices: the root lattice $\Gamma=\bigoplus_{i \in I} \mathbf{Z} \alpha_{i}$ and another lattice $L=\bigoplus_{j \in J}\left(\mathbf{Z} \alpha_{j}^{+} \oplus \mathbf{Z} \alpha_{j}^{-}\right)$with free basis $\left\{\alpha_{j}^{+}, \alpha_{j}^{-} \mid j \in J\right\}$ satisfying $\left(\alpha_{i}^{ \pm}, \alpha_{j}^{ \pm}\right)=0$ and $\left(\alpha_{i}^{+}, \alpha_{j}^{-}\right)=\delta_{i j}$. Then if $i \in I, i \notin J$ the usual vertex operator $X\left(\alpha_{i}, z\right)$ has moments tied to the basic generator $e_{i}$ while those of $X\left(-\alpha_{i}, z\right)$ are tied to $(-1)^{\left(\alpha_{i}, \alpha_{i}\right) / 2} f_{i}$. For the case of imaginary roots, if $j \in J$, then vertex operator $X^{\left(\alpha_{j}^{+}\right)}\left(\alpha_{j}, z\right)$ has moments tied to $e_{j}$ while those of $X^{\left(\alpha_{j}^{-}\right)}\left(-\alpha_{j}, z\right)$ have moments tied to $(-1)^{\left(\alpha_{j}, \alpha_{j}\right) / 2} f_{j}$. Of course, here we must also use a generalized Heisenberg Lie algebra corresponding to the lattice $L$ and we need to use the combinatorial function $\zeta_{n}(k):=\left(\begin{array}{c}k-\frac{n}{2} \\ 1-n\end{array}\right)$ for $k \in \mathbf{Z}, n \in-2 \mathbf{Z}$ to define this. As Lemma 3.38 and Corollary 3.39 show this is exactly what we need to get the commutator $\left[X^{\left(\alpha_{j}^{+}\right)}\left(\alpha_{j}, z\right), X^{\left(\alpha_{j}^{-}\right)}\left(-\alpha_{j}, z\right)\right]$ to equal the correct operator. The basic computational fact we need about the power series which arise in taking commutators of our vertex operators is in Theorem 3.22 while Proposition 3.32 deals with the basic form of what a $k$-fold commutator, $\left[X^{\left(\beta_{k}\right)}\left(\gamma_{k}, z_{k}\right), \cdots, X^{\left(\beta_{1}\right)}\left(\gamma_{1}, z_{1}\right)\right]$ looks like. Our first generalization of the Goddard-Olive result appears in Corollary 3.46 and deals with the case when the above $k$-fold commutator satisfies $\left(\beta_{i}, \beta_{j}\right)=0$ if $i \neq j$. We then go on to use this to prove Corollary 3.52 which is our full generalization of the Goddard-Olive result. We close this section by showing how this result lets us define a non-trivial representation of $\mathcal{L}(A)$. It is worth noting that the very existence of such a representation leads one to think the definition of $\mathcal{L}(A)$ is indeed very natural.

Since our matrix $A$ may be an I.M matrix we of course recover the results of EMY] on the existence of such a representation. In fact, if the reader is just interested in this case then our results become quite simple since then $J=\emptyset$ and one sees that our results are just those of [EMY] except we have put them into the formalism of [FLM] rather then using the residue calculus. Indeed, one of the starting points of this work was to do just this. Also, the matrix $A$ may define a Borcherds Lie algebra so that we obtain a general existence result about vertex representations for such algebras when the defining matrix is symmetric and has even integers on the diagonal.

\section{BASIC CONSTRUCtion AND PROperties of the LIE Algebras}

In this section we give the definition of the Lie algebras $\mathcal{G}(A)$ using generators and relations and establish some of their basic properties. Recall that a real matrix $\left(a_{i j}\right)_{i, j \in I}$ is symmetrizable if there exist positive real numbers $\epsilon_{i}, i \in I$ satisfying

$$
a_{i j} \epsilon_{j}=a_{j i} \epsilon_{i}, \quad \text { for all } i, j \in I .
$$

Definition 2.1. Let $I$ be a (finite or) countable index set. A matrix $A=\left(a_{i j}\right)_{i, j \in I}$ with real entries is admissible if it is symmmetrizable and satisfies the following two conditions:

(1) If $i \in I$ and $a_{i i}>0$, then $a_{i i}=2$,

(2) If $a_{i i}=2$ for $i \in I$, then $a_{i j} \in \mathbf{Z}$ for all $j \in I$. 
Notice that since an admissible matrix $A=\left(a_{i j}\right)_{i, j \in I}$ is symmetrizable, then we have

$$
\begin{gathered}
a_{i j}=0 \text { if and only if } a_{j i}=0, \\
a_{i j}<0 \text { if and only if } a_{j i}<0, \\
a_{i j}>0 \text { if and only if } a_{j i}>0 .
\end{gathered}
$$

Thus, if $a_{i j} \leq 0$ for all $i, j \in I$ with $i \neq j$, then an admissible matrix $A$ is just the type of matrix one uses to begain the construction of a Borcherds Lie algebra (see [Bo1]-Bo3, JLW], JJ1]). On the other hand, if $a_{i i}=2$ for all $i \in I$, then an admissible matrix is just a symmetrizable G.I.M. matrix in the sense of Slodowy (see [Sl1], [Sl2, [Be]). In this sense an admissible matrix $A$ is simultaneously a generalization of both of these cases. Also, note that in condition (1) in the above definition we have taken the usual normalization $a_{i i}=2$ when $a_{i i} \geq 0$.

We now define the Lie algebras associated to these matrices. We always work over the complex field $\mathbf{C}$.

Definition 2.3. Let $A=\left(a_{i j}\right)_{i, j \in I}$ be an admissible matrix. The Lie algebra $\mathcal{G}(A)$ associated to $A$ is defined to be the Lie algebra generated by elements $\left\{e_{i}, f_{i}, h_{i}\right\}_{i \in I}$ satisfying the following relations: $h_{i}$.

(R1) For all $i, j \in I,\left[h_{i}, h_{j}\right]=0,\left[h_{i}, e_{j}\right]=a_{i j} e_{j},\left[h_{i}, f_{j}\right]=-a_{i j} f_{j}$, and $\left[e_{i}, f_{i}\right]=$

(R2) For $i, j \in I$ with $i \neq j$ satisfying $a_{i j} \leq 0,\left[e_{i}, f_{j}\right]=0$ and, if $a_{i i}>0$, then $\left(\operatorname{ad} e_{i}\right)^{1-a_{i j}} e_{j}=0,\left(\operatorname{ad} f_{i}\right)^{1-a_{i j}} f_{j}=0$.

(R3) For $i, j \in I$ with $i \neq j$ satisfying $a_{i j}=0$ and $a_{i i} \leq 0,\left[e_{i}, e_{j}\right]=0$ and $\left[f_{i}, f_{j}\right]=0$.

(R4) For $i, j \in I$ with $i \neq j$ satisfying $a_{i j}>0,\left[e_{i}, e_{j}\right]=0,\left[f_{i}, f_{j}\right]=0$ and, if $a_{i i}>0$, then $\left(\operatorname{ad} e_{i}\right)^{1+a_{i j}} f_{j}=0,\left(\operatorname{ad} f_{i}\right)^{1+a_{i j}} e_{j}=0$.

Notice that relations (R1), (R2) and (R3) are just those used to define a Borcherds Lie algebra so if $a_{i j} \leq 0$ for all $i, j \in I$ with $i \neq j$, then $\mathcal{G}(A)$ is a Borcherds Lie algebra. Also, if $a_{i i}=2$ for all $i \in I$, then the relations above are just those defining a G.I.M. algebra of Slodowy. Of course, if $A$ satisfies both $a_{i j} \leq 0$ for all $i, j \in I$ with $i \neq j$ and $a_{i i}=2$ for all $i \in I$, then $A$ is a (possibly infinite) Cartan matrix and $\mathcal{G}(A)$ is nothing but the Kac-Moody Lie algebra attached to $A$. As special cases, if $A$ is a non-zero $1 \times 1$ matrix, then it is easy to see that $\mathcal{G}(A)$ is isomorphic to the Lie algebra $s l_{2}(\mathbf{C})$, while if $A=(0)$ is the $1 \times 1$ zero matrix, then $\mathcal{G}(A)$ is the three-dimensional Heisenberg Lie algebra. If $A$ is the $n \times n$ zero matrix, then $\mathcal{G}(A)$ is generated by elements $e_{i}, f_{i}, h_{i}, 1 \leq i \leq n$ which satisfy

$$
\left[e_{i}, f_{j}\right]=\delta_{i j} h_{i}, \quad\left[e_{i}, e_{j}\right]=0=\left[f_{i}, f_{j}\right], \quad 1 \leq i, j \leq n
$$

and $h_{1}, \ldots, h_{n}$ are central. Thus, $\mathcal{G}(A)$ is just a Lie algebra of dimension $3 n$ whose derived algebra is its $n$-dimensional center.

We say $A$ is indecomposable if whenever $I=I_{1} \cup I_{2}$ is a disjoint union and for all $i \in I_{1}, j \in I_{2}$ we have $a_{i j}=0$, then either $I_{1}$ or $I_{2}$ is empty. Note that if $I=I_{1} \cup I_{2}$ is a non-trivial disjoint union satisfying $a_{i j}=0$ whenever $i \in I_{1}, j \in I_{2}$, then letting $A_{k}=\left(a_{i j}\right)_{i, j \in I_{k}}, k=1,2$ we get (rearranging the rows and columns of A) that

$$
A=\left(\begin{array}{cc}
A_{1} & 0 \\
0 & A_{2}
\end{array}\right),
$$


and hence the usual argument shows that $\mathcal{G}(A) \cong \mathcal{G}\left(A_{1}\right) \oplus \mathcal{G}\left(A_{2}\right)$. For this reason we will restrict our attention to indecompossable admissible matrices $A$. Throughout, $A$ will denote an admissible matrix unless specified otherwise.

Our next goal is to introduce (as Slodowy does for G.I.M. matrices) the double of the matrix $A$ which we will call $\mathcal{D}(A)$ and then to study the relation between the algebras attached to $A$ and $\mathcal{D}(A)$.

Definition 2.4. Let $A=\left(a_{i j}\right)_{i, j \in I}$ be an indecomposable admissible matrix with index set $I$. Let $\tilde{I}=\{\tilde{i} \mid i \in I\}$ be a disjoint copy of $I$ and define the double of $A$, denoted $\mathcal{D}(A)$, as follows. $\mathcal{D}(A)=\left(b_{r s}\right)_{r, s \in I \cup \tilde{I}}$ where

(1) $b_{i i}=b_{\tilde{i} \tilde{i}}=a_{i i}$ for $i \in I$.

(2) $b_{i \tilde{i}}=b_{\tilde{i} i}=0$ for $i \in I$.

(3) If $i, j \in I, i \neq j$ and $a_{i j} \leq 0$, then we let $b_{i j}=b_{\tilde{i} \tilde{j}}=a_{i j}$ and $b_{i \tilde{j}}=b_{\tilde{i} j}=0$.

(4) If $i, j \in I, i \neq j$ and $a_{i j}>0$, then we let $b_{i j}=b_{\tilde{i} \tilde{j}}=0$ and $b_{i \tilde{j}}=b_{\tilde{i} j}=-a_{i j}$.

Thus, the double of an indecomposable admissible matrix $A$ is again admissible (but not necessarily indecomposable) and, in fact, has all of its off diagonal entries non-positive so that it is a matrix of Borcherds type. Hence $\mathcal{G}(\mathcal{D}(A))$ is a Borcherds Lie algebra. We denote the canonical generators of $\mathcal{G}(\mathcal{D}(A))$ by $E_{i}, E_{\tilde{i}}, F_{i}, F_{\tilde{i}}, H_{i}, H_{\tilde{i}}$, $i \in I$.

Remark 2.5. Clearly, if we do a permutation of the rows of $A$ together with the same permutation of its columns to get a matrix $A^{\prime}$, then $\mathcal{G}(A)$ and $\mathcal{G}\left(A^{\prime}\right)$ are isomorphic. Similarly, if we let $A^{\prime}$ be the matrix obtained from $A$ by first changing the sign of all entries in the $i$ th row and then the sign of all entries in the $i$ th column (so the sign of $a_{i i}$ does not change), then again $\mathcal{G}(A) \cong \mathcal{G}\left(A^{\prime}\right)$. If $A^{\prime}$ arises from $A$ by a sequence of such changes, we say $A$ and $A^{\prime}$ are equivalent.

We extend the notation $\tilde{i}$ by defining $\tilde{\tilde{i}}=i$ if $i \in I$ so that $k \rightarrow \tilde{k}$ is a period 2 mapping of $I \cup \tilde{I}$ to itself.

Lemma 2.6. Let $A=\left(a_{i j}\right)_{i, j \in I}$ be an admissible indecomposable matrix. Then $\mathcal{D}(A)$ is either indecomposable or is equivalent to

$$
D^{\prime}=\left(\begin{array}{cc}
D_{1} & 0 \\
0 & D_{2}
\end{array}\right)
$$

where both $D_{1}, D_{2}$ are of Borcherds type and we have the Borcherds Lie algebras $\mathcal{G}\left(D_{1}\right)$ and $\mathcal{G}\left(D_{2}\right)$ are isomorphic to each other and also to $\mathcal{G}(A)$.

Proof. As usual, one says that two indicies $i$ and $j$ are connected if and only if they are equal or there is a sequence of indicies $i_{1}, \ldots, i_{t}$ with $i=i_{1}, j=i_{t}$ and $a_{i_{k-1} i_{k}} \neq 0$ for $k=2, \ldots, t$. Thus, $A$ is indecomposable if and only if all indicies are connected. Fix $i_{0} \in I$ and note that $A$ indecomposable implies that for all $i \in I$ either $i$ or $\tilde{i}$ is connected to $i_{0}$, hence either $\mathcal{D}(A)$ is indecomposable or $I \cup \tilde{I}$ has two connected components, one containing $i_{0}$ and the other containing $\tilde{i}_{0}$. Let the component containing $i_{0}$ be denoted $U$ so that $\tilde{U}=\{\tilde{u} \mid u \in U\}$ is the component containing $\tilde{i}_{0}$. Assume $\mathcal{D}(A)$ is not indecomposable. Then for $i \in I \cup \tilde{I}$ we have $i \in U$ if and only if $\tilde{i} \in \tilde{U}$ and if $D_{1}$ (respectively $D_{2}$ ) is the submatrix of $\mathcal{D}(A)$ attached to $U$ (respectively $\tilde{U})$, then we have $D_{1}$ and $D_{2}$ are equivalent and so $\mathcal{G}\left(D_{1}\right) \cong \mathcal{G}\left(D_{2}\right)$ and since $\mathcal{D}(A)$ is equivalent to $\left(\begin{array}{cc}D_{1} & 0 \\ 0 & D_{2}\end{array}\right)$, then $\mathcal{G}(\mathcal{D}(A)) \cong \mathcal{G}\left(D_{1}\right) \oplus \mathcal{G}\left(D_{2}\right)$.

Moreover, still assuming $\mathcal{D}(A)$ is not indecomposable we have $I \cup \tilde{I}$ equals to the union of $U$ and $\tilde{U}$ and, moreover, the intersection $U \cap \tilde{U}$ is empty. Also, 
$U=(I \cap U) \cup(\tilde{I} \cap U)$. It follows that if $i \in I \cap U$ and $\tilde{j} \in \tilde{I} \cap U$ for some $j \in I$, then $a_{i j} \geq 0$, otherwise, both $j$ and $\tilde{j}$ would be connected to $i_{0}$ which then implies $\mathcal{D}(A)$ is indecomposable. It follows from this, that by making a sequence of sign changes and permutations of rows and the corresponding columns that $A$ is equivalent to $D_{1}$ so that $\mathcal{G}(A) \cong \mathcal{G}\left(D_{1}\right) \cong \mathcal{G}\left(D_{2}\right)$ as required.

Following the G.I.M. case we make the following definition:

Definition 2.7. We say the indecomposable admissible matrix $A$ is oriented if its double $\mathcal{D}(A)$ has two indecomposable components. Otherwise, when $\mathcal{D}(A)$ is indecomposable, we say $A$ is unoriented.

Remark 2.8. Assume $A$ is oriented so that $\mathcal{D}(A)=\left(\begin{array}{cc}D_{1} & 0 \\ 0 & D_{2}\end{array}\right)$ and $\mathcal{G}(\mathcal{D}(A)) \cong$ $\mathcal{G}\left(D_{1}\right) \oplus \mathcal{G}\left(D_{2}\right)$ as above where also $\mathcal{G}(A) \cong \mathcal{G}\left(D_{1}\right) \cong \mathcal{G}\left(D_{2}\right)$. Let $I \cup \tilde{I}=U \cup \tilde{U}$ where $U$ and $\tilde{U}$ are the two connected components. Then the map $i \rightarrow \tilde{i}$ of $I \cup \tilde{I}$ to itself gives rise to an automorphism $\tau$ of $\mathcal{G}(\mathcal{D}(A))$ to itself where

$$
\tau\left(X_{i}\right)=X_{\tilde{i}} \text { for } i \in I \cup \tilde{I}, X \in\{E, F, H\} .
$$

Clearly, $\tau$ is of period 2 and commutes with the automorphism $\eta$ of $\mathcal{G}(\mathcal{D}(A))$ defined by

$$
\eta\left(E_{i}\right)=F_{i}, \eta\left(F_{i}\right)=E_{i}, \eta\left(H_{i}\right)=-H_{i}, \quad i \in I \cup \tilde{I} .
$$

Thus, letting $\sigma=\tau \circ \eta$ we see that $\sigma$ is an automorphism of $\mathcal{G}(\mathcal{D}(A))$ of order 2 which satisfies that

$$
\sigma\left(E_{i}\right)=F_{\tilde{i}}, \sigma\left(F_{i}\right)=E_{\tilde{i}}, \sigma\left(H_{i}\right)=-H_{\tilde{i}}, \quad i \in I \cup \tilde{I} .
$$

Let $\mathcal{G}(\mathcal{D}(A))^{\sigma}$ be the fixed points of $\mathcal{G}(\mathcal{D}(A))$ under $\sigma$. Clearly, we have $\mathcal{G}(\mathcal{D}(A))^{\sigma}=$ $\left\{x+\sigma(x) \mid x \in \mathcal{G}\left(D_{1}\right)\right\}$, so that it follows that $\mathcal{G}(\mathcal{D}(A))^{\sigma} \cong \mathcal{G}\left(D_{1}\right)$ which we know is isomorphic to $\mathcal{G}(A)$. A particular isomorphism of $\mathcal{G}(A)$ with $\mathcal{G}(\mathcal{D}(A))^{\sigma}$ is given by

$$
\begin{aligned}
e_{i} & \rightarrow E_{i}+F_{\tilde{i}}, \\
f_{i} & \rightarrow F_{i}+E_{\tilde{i}}, \\
h_{i} & \rightarrow H_{i}-H_{\tilde{i}},
\end{aligned}
$$

for $i \in I$. Thus, in this case, $\mathcal{G}(A)$ is isomorphic to a subalgebra of fixed points of a period 2 automorphism of $\mathcal{G}(\mathcal{D}(A)$ ), and hence is 'inside of' $\mathcal{G}(\mathcal{D}(A)$ ). Our next goal is to show this remains true in the unoriented case as well. The argument is almost exactly the same as in [Be] so we will be brief.

Let $A$ be an arbitrary indecomposable admissible matrix. Clearly, the mapping $\sigma$ defined on generators by saying

$$
\sigma\left(E_{i}\right)=F_{\tilde{i}}, \sigma\left(F_{i}\right)=E_{\tilde{i}}, \sigma\left(H_{i}\right)=-H_{\tilde{i}}, \quad i \in I \cup \tilde{I},
$$

is a period two automorphism on $\mathcal{G}(\mathcal{D}(A))$ even in the case when $A$ is unoriented.

Let $\mathcal{G}(\mathcal{D}(A))=\mathcal{G}$ and $\mathcal{S}=\{x \in \mathcal{G} \mid \sigma(x)=x\}$. Define elements $x_{i}, z_{i}, i \in I \cup \tilde{I}$ of $\mathcal{S}$ as follows:

$$
x_{i}=E_{i}+F_{\tilde{i}}, \quad z_{i}=H_{i}-H_{\tilde{i}}, \quad i \in I \cup \tilde{I} .
$$

If $\mathcal{G}=\mathcal{G}^{+} \oplus \mathcal{H} \oplus \mathcal{G}^{-}$is the usual triangular decomposition of the Borcherds algebra, then in fact $\mathcal{S}=\left\{x+\sigma(x) \mid x \in \mathcal{H} \oplus \mathcal{G}^{+}\right\}$. We will show (in Theorem 2.11) that $\mathcal{S}$ is isomorphic to $\mathcal{G}(A)$. First we construct a filtration of $\mathcal{S}$. Let

$$
\mathcal{S}^{-1}=\{0\}, \quad \mathcal{S}^{0}=\sum_{i \in I} \mathbf{C} z_{i}, \quad \mathcal{S}^{1}=\mathcal{S}^{0}+\sum_{i \in I} \mathbf{C} x_{i},
$$


in general, define $\mathcal{S}^{n}$ for $n \geq 2$ to be the sum of $\mathcal{S}^{n-1}$ and the $\mathbf{C}$-linear span of all the elements $\left\{\left[x_{j_{1}}, x_{j_{2}}, \cdots, x_{j_{n}}\right]\left(=\left[\left[\left[x_{j_{1}}, x_{j_{2}}\right], x_{j_{3}}\right], \cdots\right]\right) \mid j_{k} \in I \cup \tilde{I}\right\}$. We have

$$
\begin{gathered}
\mathcal{S}^{n}=\mathcal{S}^{n-1}+\left[\mathcal{S}^{n-1}, \mathcal{S}^{1}\right], \quad(0)=\mathcal{S}^{-1} \subset \mathcal{S}^{0} \subset \mathcal{S}^{1} \subset \cdots \subset \mathcal{S}^{n}, \\
{\left[\mathcal{S}^{n}, \mathcal{S}^{m}\right] \subset \mathcal{S}^{n+m} .}
\end{gathered}
$$

Lemma 2.10. The subalgebra $\mathcal{S}$ of the Borcherds algebra $\mathcal{G}$ is generated by the elements $\left\{x_{i}, z_{i} \mid i \in I \cup \tilde{I}\right\}$. In particular, (2.9) defines a filtration of $\mathcal{S}$. Moreover, for $n \geq 1$ and any indices $j_{k} \in I \cup \tilde{I}$ we have

$$
\left[E_{j_{1}}, \cdots, E_{j_{n}}\right]+\sigma\left(\left[E_{j_{1}}, \cdots, E_{j_{n}}\right]\right)=\left[x_{j_{1}}, \cdots, x_{j_{n}}\right] \bmod \mathcal{S}^{n-1} .
$$

In particular, if $n=b_{k j}+1$, then $\left(a d x_{k}\right)^{n} x_{j}=0 \bmod \mathcal{S}^{n}$.

Proof. The proof in $[\mathrm{Be}]$ carries over to this case without change. One shows first that

$$
\left[E_{i}, E_{j}\right]+\sigma\left(\left[E_{i}, E_{j}\right]\right)=\left[x_{i}, x_{j}\right]-\delta_{i, \tilde{j}} z_{i}
$$

and the result follows by induction and the fact that $\mathcal{S}=\left\{x+\sigma(x) \mid x \in \mathcal{H} \oplus \mathcal{G}^{+}\right\}$.

The generators of $\mathcal{G}^{+}$are the elements $E_{i}$ for $i \in I \cup \tilde{I}$. We let $n \geq 1$ and let $\mathcal{G}_{n}$ be the span of the elements $\left[E_{i_{1}}, \cdots, E_{i_{n}}\right]$ for $i_{j} \in I \cup \tilde{I}$. Clearly, then we have $\mathcal{G}^{+}=\bigoplus_{n \geq 1} \mathcal{G}_{n}$ is a $\mathbf{Z}$-grading of $\mathcal{G}^{+}$.

Let $S=\bigoplus_{n \geq 0} S_{n}$ where $S_{n}=\mathcal{S}^{n} / \mathcal{S}^{n-1}$ so that $S$ is the associated graded Lie algebra of the filtered Lie algebra $\mathcal{S}$. We let $S^{+}=\bigoplus_{n \geq 1} S_{n}$ so $S^{+}$is a subalgebra of $S$.

Lemma 2.11. For $n \geq 1$ we have $\operatorname{dim} S_{n}=\operatorname{dim} \mathcal{G}_{n}$.

Proof. We know that $\mathcal{S}^{n}=\left\{x+\sigma(x) \mid x \in \mathcal{G}_{n}\right\}+\mathcal{S}^{n-1}$ for $n \geq 1$ so the map of $\mathcal{G}_{n}$ to $S_{n}$ given by $x \mapsto(x+\sigma(x))+\mathcal{S}^{n-1}$ is surjective. Also, if $x \in \mathcal{G}_{n}$ but $x+\sigma(x) \in \mathcal{S}^{n-1}$, then, since $\sigma(x) \in \mathcal{G}^{-}$, there is some $y \in \bigoplus_{k=1}^{n-1} \mathcal{G}_{k}$ for which $x=y$. This forces $x=y=0$ since we have the direct $\operatorname{sum} \mathcal{G}^{+}=\bigoplus_{n \geq 1} \mathcal{G}_{n}$.

We are now in a position to establish that the algebra $\mathcal{G}(A)$ is indeed isomorphic to a subalgebra of the Borcherds algebra $\mathcal{G}(\mathcal{D}(A))$ in all cases.

Theorem 2.12. Let $A$ be an indecomposable admissible matrix. Then $\mathcal{G}(A)$ is isomorphic to the subalgebra $\mathcal{G}(\mathcal{D}(A))^{\sigma}$ of $\mathcal{G}(\mathcal{D}(A))$ consisting of fixed points of the period two automorphism $\sigma$ of $\mathcal{G}(\mathcal{D}(A))$.

Proof. We restrict ourself to the case when $A$ is unoriented since in Remark 2.8 we have dealt with the oriented case. It is straightforward to see that the elements $E_{i}+F_{\tilde{i}}, F_{i}+E_{\tilde{i}}, H_{i}-H_{\tilde{i}}$ for $i \in I$ satisfy the relations (R1) through (R4). For example, if $i, j \in I$, we have

$$
\left[H_{i}-H_{\tilde{i}}, E_{j}+F_{\tilde{j}}\right]=\left(b_{i j}-b_{\tilde{i} j}\right) E_{j}+\left(-b_{i \tilde{j}}+b_{\tilde{i} j}\right) F_{\tilde{j}}=a_{i j}\left(E_{i}+F_{\tilde{j}}\right),
$$

because $b_{i j}-b_{\tilde{i} j}=b_{i \tilde{j}}-b_{i \tilde{j}}=a_{i j}$ in all cases. To check another case we assume $i, j \in I, i \neq j$ and $a_{i j}>0$. Then

$$
\left[E_{i}+F_{\tilde{i}}, E_{j}+F_{\tilde{j}}\right]=\left[E_{i}, E_{j}\right]+\left[F_{\tilde{i}}, F_{\tilde{j}}\right]=0
$$

since $a_{i j}>0$ implies $b_{i j}=0=b_{\tilde{i} \tilde{j}}$, while we have, if $a_{i i}>0$, that

$$
\operatorname{ad}\left(E_{i}+F_{\tilde{i}}\right)^{1+a_{i j}}\left(F_{j}+E_{\tilde{j}}\right)=\left(\operatorname{ad} E_{i}\right)^{1+a_{i j}} E_{\tilde{i}}+\left(\operatorname{ad} F_{\tilde{j}}\right)^{1+a_{i j}} F_{j}=0
$$


since here $b_{i j}=b_{\tilde{i} \tilde{j}}=0$ but $b_{\tilde{i} j}=b_{i \tilde{j}}=-a_{i j}$ and we are in a Borcherds algebra and $b_{i i}=a_{i i}>0$. It follows that there is a homomorphism $\varphi: \mathcal{G}(A) \rightarrow \mathcal{G}(\mathcal{D}(A))^{\sigma}=\mathcal{S}$ satisfying

$$
\varphi\left(e_{i}\right)=E_{i}+F_{\tilde{i}}, \quad \varphi\left(f_{i}\right)=F_{i}+E_{\tilde{i}}, \quad \varphi\left(h_{i}\right)=H_{i}-H_{\tilde{i}}, \quad i \in I .
$$

Moreover, Lemma 2.10 implies that $\varphi$ is surjective, so we only need to show $\varphi$ is injective.

Towards this end we introduce a filtration on $\mathcal{G}(A)$ as follows. Let $\mathcal{L}=\mathcal{G}(A)$ and let $\mathcal{L}^{-1}=(0)$, and $\mathcal{L}^{0}$ equal the span of the elements $e_{i}, f_{i}$ for $i \in I$, and inductively we define $\mathcal{L}^{n}$ to be $\mathcal{L}^{n-1}$ plus the span of all elements $\left[x_{j_{1}}, \cdots, x_{j_{n}}\right]$ where $x_{j_{k}} \in\left\{e_{i}, f_{i} \mid i \in I\right\}$. Then we have for $n, m \geq 0$

$$
\left[\mathcal{L}^{n}, \mathcal{L}^{m}\right] \subseteq \mathcal{L}^{n+m}, \quad \mathcal{L}^{-1} \subseteq \mathcal{L}^{0} \subseteq \mathcal{L}^{1} \subseteq \cdots,
$$

and $\mathcal{L}=\bigcup_{n \geq 0} \mathcal{L}^{n}$, so $\mathcal{L}$ is filtered by the spaces $\mathcal{L}^{n}$. Let $L_{n}=\mathcal{L}^{n} / \mathcal{L}^{n-1}$ for $n \geq 0$ so that $L=\bigoplus_{n>0} L_{n}$ is the associated graded algebra to the filtered Lie algebra $\mathcal{L}$. Let $L^{+}=\bigoplus_{n>1} L_{n}$. This is a subalgebra of $L$.

Note that $\varphi: \mathcal{L} \rightarrow \mathcal{S}$ is a filtered homomorphism in the sense that $\varphi\left(\mathcal{L}^{n}\right)=\mathcal{S}^{n}$ for all $n \geq 0$ so that $\varphi$ induces a graded Lie algebra homomorphism, $\psi$, of $L$ to $S$ defined on $L_{n}$ by saying, if $x \in \mathcal{L}^{n}$, then

$$
\psi\left(x+\mathcal{L}^{n-1}\right)=\varphi(x)+\mathcal{S}^{n-1},
$$

and then extending this to all of $L$ using additivity. Clearly, $\psi$ is a surjective Lie algebra homomorphism and $\psi\left(L_{n}\right)=S_{n}$ for all $n \geq 0$. Moreover, it is well-known that if $\psi$ is injective, then $\varphi$ is injective so it is enough to show $\psi$ is injective. Also, clearly $\psi$ restricted to $L_{0}$ is injective since $\psi\left(h_{i}\right)=H_{i}-H_{\tilde{i}}$ for $i \in I$ and we know that elements $H_{i}-H_{\tilde{i}}, i \in I$ are linearly independent in our Borcherds algebra $\mathcal{G}(\mathcal{D}(A))$. Thus it is enough to show $\psi$ restricted to $L_{n}$ is injective for all $n \geq 1$ and since we know $\psi$ is onto we have that, by Lemma 2.11,

$$
\operatorname{dim} L_{n} \geq \operatorname{dim} S_{n}=\operatorname{dim} \mathcal{G}_{n}, \quad \text { for } n \geq 1 .
$$

Thus, it is enough to show that $\operatorname{dim} \mathcal{G}_{n} \geq \operatorname{dim} L_{n}$ for all $n \geq 1$ to conclude that $\varphi$ is injective.

Recall that by the Gabber-Kac theorem for the Borcherds algebra $\mathcal{G}=\mathcal{G}(\mathcal{D}(A))$ one knows that $\mathcal{G}^{+}$is the factor algebra of the free Lie algebra on the generators $E_{i}, i \in I \cup \tilde{I}$ factored by the ideal $\mathcal{J}$ consisting of the following elements:

$$
\left(\operatorname{ad} E_{j}\right)^{1-b_{j k}} E_{k}, \quad \text { for } k, j \in I \cup \tilde{I}, \quad k \neq j, \quad b_{j j}>0 ;
$$

and the elements

$$
\left[E_{j}, E_{k}\right], \quad \text { for } k, j \in I \cup \tilde{I}, \quad k \neq j, \quad b_{j j} \leq 0 \text { and } b_{j k}=0 .
$$

We let $\tau:\left\{E_{i} \mid i \in I \cup \tilde{I}\right\} \rightarrow L^{+}$be defined by $\tau\left(E_{i}\right)=e_{i}+\mathcal{L}^{0}, \tau\left(E_{\tilde{i}}\right)=f_{i}+\mathcal{L}^{0}$ for $i \in I$. It is easy to check that these elements satisfy the relations (2.13) and (2.14). For example, we know $\left[E_{i}, E_{\tilde{i}}\right]$ is one of the element defining $\mathcal{J}$. Indeed, $b_{i \tilde{i}}=0$ for any $i \in I$ so $\left[E_{i}, E_{\tilde{i}}\right]$ is of type (2.13) if $b_{i i}>0$ and of type (2.14) if $b_{i i} \leq 0$. In either case we have $\left[\tau\left(E_{i}\right), \tau\left(E_{\tilde{i}}\right)\right]=\left[e_{i}+\mathcal{L}^{0}, f_{i}, \mathcal{L}^{0}\right]=h_{i}+\mathcal{L}^{1}=0$ in $L^{+}$. To check another case, suppose $b_{j j}>0, j \in I, \tilde{k} \in \tilde{I}$ (so $k \in I$ ) and $a_{j k}>0$. Then $b_{j \tilde{k}}=-a_{j k}$ and we know $a_{j j}=b_{j j}>0$ so $\left(\operatorname{ad} E_{j}\right)^{1-b_{j \tilde{k}}} E_{\tilde{k}}=\left(\operatorname{ad} E_{j}\right)^{1+a_{j k}} E_{\tilde{k}}$ is one of the elements defining the ideal $\mathcal{J}$. Now 


$$
\left(\operatorname{ad} \tau\left(E_{j}\right)\right)^{1+a_{j k}} \tau\left(E_{\tilde{k}}\right)=\left(\operatorname{ad}\left(e_{i}+\mathcal{L}^{0}\right)\right)^{1+a_{j k}}\left(f_{k}+\mathcal{L}^{0}\right)=\left(\operatorname{ad} e_{j}\right)^{1+a_{j k}} f_{k}+\mathcal{L}^{2+a_{j k}},
$$

but by relation (R4) we have (since $a_{j j}>0$ and $a_{j k}>0$ ) that $\left(\operatorname{ad} e_{j}\right)^{1+a_{j k}} f_{k}=0$ in $\mathcal{L}=\mathcal{G}(A)$. The other cases are similar to check.

It follows that $\tau$ extends to a homomorphism of $\mathcal{G}^{+}$to $\mathcal{L}^{+}$which is graded in the sense that $\tau\left(\mathcal{G}_{n}\right)=L_{n}$ for $n \geq 1$ and, moreover, $\tau$ is onto as $L^{+}$is generated by the elements $e_{i}+\mathcal{L}^{0}, f_{i}+\mathcal{L}^{0}, i \in I$.

It follows from this that $\operatorname{dim} \mathcal{G}_{n} \geq \operatorname{dim} L_{n}$ for all $n \geq 1$, thereby proving our result.

Remark 2.15. The above proof shows that $\tau: \mathcal{G}^{+} \rightarrow L^{+}$is an isomorphism of $\mathcal{G}^{+}$ onto $L^{+}$so we actually determined the structure of the associated graded algebra to the filtered Lie algebra $\mathcal{G}(A)$.

We conclude this section by showing how the algebras $\mathcal{G}(A)$ are related to the concept of affinizing Lie algebras. For this, recall, from [FLM], that if $\mathcal{L}$ is a Lie algebra with a symmetric bilinear form which is invariant, then letting $\mathbf{C}\left[t, t^{-1}\right]$ be the ring of finite Laurent polynomials one considers the vector space

$$
\hat{\mathcal{L}}=\mathcal{L} \otimes \mathbf{C}\left[t, t^{-1}\right] \oplus \mathbf{C} c,
$$

where $\mathbf{C} c$ is a one-dimensional space. The product on $\hat{\mathcal{L}}$ is determined by saying $c$ is central in $\hat{\mathcal{L}}$ and for $x, y \in \mathcal{L}, n, m \in \mathbf{Z}$ one has

$$
\left[x \otimes t^{m}, y \otimes t^{n}\right]=[x, y] \otimes t^{m+n}+(x, y) m \delta_{m+n, 0} c .
$$

Then $\hat{\mathcal{L}}$ is a Lie algebra which is called the affine Lie algebra associated to the pair $(\mathcal{L},(\cdot, \cdot))$. Note $\mathcal{L} \otimes 1$ is a subalgebra of $\hat{\mathcal{L}}$ which is isomorphic to $\mathcal{L}$.

Now we assume that there is an admissible indecomposable matrix $A=\left(a_{i j}\right)_{i, j \in I}$ for which there is a surjective homomorphism $\varphi: \mathcal{G}(A) \rightarrow \mathcal{L}$. Moreover, we assume there is an index $i_{0} \in I$ for which

$$
\begin{gathered}
a_{i_{0} i_{0}}>0\left(\text { so } a_{i_{0} i_{0}}=2\right) \text { and } \\
\left(\varphi\left(e_{i_{0}}\right), \varphi\left(f_{i_{0}}\right)\right) \neq 0 .
\end{gathered}
$$

Here, for example, one might take $\mathcal{L}$ to be any of the Lie algebras $\mathcal{G}(A)$ where $(2.16)$ and (2.17) hold with $\varphi$ being the identity mapping, so in this case $\hat{\mathcal{L}}$ is nothing but the affinization of $\mathcal{G}(A)$. We are going to define a new admissible matrix $\hat{A}$ and a surjective homomorphism $\psi: \mathcal{G}(\hat{A}) \rightarrow \hat{\mathcal{L}}$ which satisfies $\psi(x)=\varphi(x) \otimes 1$ if $x \in\left\{e_{i}, f_{i}, h_{i} \mid i \in I\right\}$.

To do this let $j_{0}$ be a new index and let $J=I \cup\left\{j_{0}\right\}$, a disjoint union. Define $b_{i j}$ for $i, j \in J$ by

$$
b_{i j}= \begin{cases}a_{i j}, & \text { if } i, j \in I, \\ a_{i_{0} j}, & \text { if } i=j_{0}, j \in I, \\ a_{i i_{0}}, & \text { if } i \in I, j=j_{0}, \\ a_{i_{0} i_{0}}, & \text { if } i=j=j_{0},\end{cases}
$$

and let $\hat{A}=\left(b_{i j}\right)_{i, j \in J}$. Clearly, $\hat{A}$ is admissible and indecomposable since $A$ is. Also, if $\hat{A}$ is oriented, then so is $A$ but it can very well be the case that $A$ is oriented but $\hat{A}$ is unoriented. (This happens, for example, if $A$ is the affine Cartan matrix of 
type $A_{1}^{(1)}$.) Define a map $\psi$ from the set of generators $\left\{e_{i}, f_{j}, h_{j} \mid j \in J\right\}$ of $\mathcal{G}(\hat{A})$ to $\hat{\mathcal{L}}$ by

$$
\begin{gathered}
\psi\left(e_{i}\right)=\varphi\left(e_{i}\right) \otimes 1, \quad \psi\left(f_{i}\right)=\varphi\left(f_{i}\right) \otimes 1, \\
\psi\left(h_{i}\right)=\varphi\left(h_{i}\right) \otimes 1, \quad \text { for } i \in I \text { and } \\
\psi\left(e_{j_{0}}\right)=\varphi\left(e_{i_{0}}\right) \otimes t, \quad \psi\left(f_{j_{0}}\right)=\varphi\left(f_{i_{0}}\right) \otimes t^{-1}, \\
\psi\left(h_{j_{0}}\right)=\varphi\left(h_{i_{0}}\right) \otimes 1+\left(\varphi\left(e_{i_{0}}\right), \varphi\left(f_{i_{0}}\right)\right) c .
\end{gathered}
$$

Our main result about affinization is the following:

Proposition 2.20. Let $\mathcal{L}$ be a Lie algebra with a symmetric invariant bilinear form and assume there $i s$ an admissible indecomposable matrix $A=\left(a_{i j}\right)_{i, j \in I}$ and a surjective homomorphism $\varphi: \mathcal{G}(A) \rightarrow \mathcal{L}$ as well as an index $i_{0} \in I$ for which (2.16) and (2.17) hold. Then there is a surjective homomorphism $\psi: \mathcal{G}(\hat{A}) \rightarrow \hat{\mathcal{L}}$ defined on the generators of $\mathcal{G}(\hat{A})$ by (2.18) and (2.19).

Proof. To show the homomorphism $\psi$ exists it is enough to show the elements in (2.18) and (2.19) satisfy the relations (R1) through (R4) for the matrix $\hat{A}=$ $\left(b_{i j}\right)_{i, j \in J}$. Now since we already have $\varphi: \mathcal{G}(A) \rightarrow \mathcal{L}$ is a surjective homomorphism, we know the relations (R1) through (R4) hold when the indicies $i, j$ come from the subset $I$ of $J$ so it is enough to check that (R1) through (R4) hold when at least one of the indicies involved is $j_{0}$. This is straightforward so we content ourselves with a few sample computations.

Clearly, we have $\left[\psi\left(h_{i}\right), \psi\left(h_{j_{0}}\right)\right]=\left[\varphi\left(h_{i}\right) \otimes 1, \varphi\left(h_{i_{0}}\right) \otimes 1+\left(\varphi\left(e_{i_{0}}\right), \varphi\left(f_{i_{0}}\right)\right) c\right]=0$ for $i \in I$. Also, for $i \in I$

$$
\left[\psi\left(h_{i}\right), \psi\left(e_{j_{0}}\right)\right]=\left[\varphi\left(h_{i}\right) \otimes 1, \varphi\left(e_{i_{0}}\right) \otimes t\right]=\left[\varphi\left(h_{i}\right), \varphi\left(e_{i_{0}}\right)\right] \otimes t .
$$

But $\left[\varphi\left(h_{i}\right), \varphi\left(e_{i_{0}}\right)\right]$ equals $a_{i i_{0}} \varphi\left(e_{i_{0}}\right)=b_{i j_{0}} \varphi\left(e_{i_{0}}\right)$ so we obtain

$$
\left[\psi\left(h_{i}\right), \psi\left(e_{j_{0}}\right)\right]=b_{i j_{0}}\left(\varphi\left(e_{i_{0}}\right) \otimes t\right)=b_{i j_{0}} \psi\left(e_{j_{0}}\right) \text {. }
$$

This also holds when $i=j_{0}$ and when $i=j_{0}$ and $j \in I$. Similarly, when we use $\psi\left(f_{j_{0}}\right)$ in place of $\psi\left(e_{j_{0}}\right)$ in the above we get the appropriate relations in (R1). Also, we have

$\left[\psi\left(e_{j_{0}}\right), \psi\left(f_{j_{0}}\right)\right]=\left[\varphi\left(e_{i_{0}}\right) \otimes t, \varphi\left(f_{i_{0}}\right) \otimes t^{-1}\right]=\varphi\left(h_{i_{0}}\right) \otimes 1+\left(\varphi\left(e_{i_{0}}\right), \varphi\left(f_{i_{0}}\right)\right) c=\psi\left(h_{j_{0}}\right)$.

To check some of the relations in (R2) assume $i, j \in J, i \neq j$ and $b_{i j} \leq 0$ where either $i$ or $j$ is $j_{0}$. If $i=j_{0}$, then $b_{i j}=a_{i_{0} j}$ and since $b_{i j} \leq 0$ and $a_{i_{0} i_{0}}>0$, we have $j \neq i_{0}, j \neq j_{0}$. Then

$$
\left[\psi\left(e_{i}\right), \psi\left(f_{j}\right)\right]=\left[\psi\left(e_{j_{0}}\right), \psi\left(f_{j}\right)\right]=\left[\varphi\left(e_{i_{0}}\right) \otimes t, \varphi\left(f_{j}\right) \otimes 1\right] .
$$

This is zero because $\left[\varphi\left(e_{i_{0}}\right), \varphi\left(f_{j}\right)\right]=\varphi\left(\left[e_{i_{0}}, f_{j}\right]\right)$ is zero since $a_{i_{0} j}=b_{i j} \leq 0$. Moreover, $a_{i_{0} i_{0}}>0$ implies (by (R2)) that $\left(a d e_{i_{0}}\right)^{1-a_{i_{0} j}} e_{j}=0$ so it follows that $\left(a d\left(\psi\left(e_{i}\right)\right)\right)^{1-b_{i j}} \psi\left(e_{j}\right)=0$. One argues similarly for the $f$ 's and the case when $i \in I$, $i \neq i_{0}, j=j_{0}$. (R3) is easy to check since one must have the index $i \neq i_{0}, j_{0}$ because $a_{i_{0} i_{0}}>0$.

Consider the relations of type (R4). Thus take $i, j \in J, i \neq j$ with $b_{i j}>0$ and one of $i$ or $j$ is $j_{0}$. Assume $j=j_{0}$. Then $i \in I$ and $b_{i j}=a_{i i_{0}}$ so here we may have $i=i_{0}$. If we are in this case when $i=i_{0}$, then

$$
\left[\psi\left(e_{i}\right), \psi\left(e_{j}\right)\right]=\left[\varphi\left(e_{i_{0}}\right) \otimes 1, \varphi\left(e_{i_{0}}\right) \otimes t\right]
$$


which is clearly zero while since $a_{i_{0} i_{0}}>0$ and $b_{i j}=a_{i_{0} i_{0}}=2$ so the element

$$
\left(\operatorname{ad}\left(\psi\left(e_{i}\right)\right)\right)^{1+b_{i j}} \psi\left(f_{j}\right)=\left(\operatorname{ad}\left(\varphi\left(e_{i_{0}}\right) \otimes 1\right)^{3}\left(\varphi\left(f_{i_{0}}\right) \otimes t^{-1}\right) .\right.
$$

But by $(2.17)$ we know $\left(\varphi\left(e_{i_{0}}\right), \varphi\left(f_{i_{0}}\right)\right) \neq 0$ so that $\varphi\left(e_{i_{0}}\right), \varphi\left(f_{i_{0}}\right)$ are non-zero elements in $\mathcal{L}$. It follows that the span of $\varphi\left(h_{i_{0}}\right), \varphi\left(e_{i_{0}}\right), \varphi\left(f_{i_{0}}\right)$ in $\mathcal{L}$ is isomorphic to the Lie algebra $s l_{2}(\mathbf{C})$, so, in particular, $\left(\operatorname{ad} \varphi\left(e_{i_{0}}\right)\right)^{3} \varphi\left(f_{i_{0}}\right)$ is zero. It follows that $\left(\operatorname{ad}\left(\psi\left(e_{i}\right)\right)\right)^{1+b_{i j}} \psi\left(f_{j}\right)=0$ in the case we are considering.

If we still have $i, j \in J, i \neq j$ with $b_{i j}>0, j=j_{0}$ but $i \neq i_{0}$, then it easily follows that

$$
\left[\psi\left(e_{i}\right), \psi\left(e_{j}\right)\right]=\left[\varphi\left(e_{i}\right) \otimes 1, \varphi\left(e_{i_{0}}\right) \otimes t\right]=0
$$

and, if $a_{i i}>0$, then

$$
\left(\operatorname{ad}\left(\psi\left(e_{i}\right)\right)\right)^{1+b_{i j}} \psi\left(f_{j}\right)=\left(\operatorname{ad}\left(\varphi\left(e_{i}\right) \otimes 1\right)\right)^{1+a_{i i_{0}}}\left(\varphi\left(f_{i_{0}}\right) \otimes t^{-1}\right)=0
$$

from the corresponding relation for $\mathcal{G}(A)$.

One argues in a similar way when $i \neq j, b_{i j}>0, i=j_{0}$ so that $j \in I$ and then this takes care of (R4). Thus, we have a homomorphism $\psi$ from $\mathcal{G}(\hat{A})$ to $\hat{\mathcal{L}}$.

Note that since $\left(\varphi\left(e_{i_{0}}\right), \varphi\left(f_{i_{0}}\right)\right) \neq 0$, then $c$ belongs to $\operatorname{Im} \psi$, the image of $\psi$. Clearly, we also have $\varphi\left(e_{i_{0}}\right) \otimes t^{ \pm 1}, \varphi\left(f_{i_{0}}\right) \otimes t^{ \pm 1}, \varphi\left(h_{i_{0}}\right) \otimes t^{ \pm 1}$ are in $\operatorname{Im} \psi$ and from this it follows that $\varphi\left(e_{i_{0}}\right) \otimes t^{n}, \varphi\left(f_{i_{0}}\right) \otimes t^{n}, \varphi\left(h_{i_{0}}\right) \otimes t^{n}$ are in $\operatorname{Im} \psi$ for all $n \in \mathbf{Z}$. Then, because $A$ is indecomposable, we get that $\varphi\left(e_{i}\right) \otimes t^{n}, \varphi\left(f_{i}\right) \otimes t^{n}, \varphi\left(h_{i}\right) \otimes t^{n}$ are in $\operatorname{Im} \psi$ for all $n \in \mathbf{Z}$. But $\operatorname{Im} \psi$ is a subalgebra of $\hat{\mathcal{L}}$ and clearly these elements generate $\hat{\mathcal{L}}$, because the elements $\left\{\varphi\left(e_{i}\right), \varphi\left(f_{i}\right), \varphi\left(h_{i}\right) \mid i \in I\right\}$ generate $\mathcal{L}$, so it follows that $\psi$ is surjective.

One more observation is in order.

Remark 2.21. With the situation as in the last proposition, one knows that the form $(\cdot, \cdot): \mathcal{L} \times \mathcal{L} \rightarrow \mathbf{C}$ can be extended to a symmetric invariant bilinear form on $\hat{\mathcal{L}}$ which we also denote by $(\cdot, \cdot)$ and which satisfies

$$
\begin{gathered}
(c, \hat{\mathcal{L}})=(0)=(\hat{\mathcal{L}}, c), \text { and } \\
\left(x \otimes t^{n}, y \otimes t^{m}\right)=(x, y) \delta_{n+m, 0}
\end{gathered}
$$

for $x, y \in \mathcal{L}, m, n \in \mathbf{Z}$. Thus, we have $\hat{\mathcal{L}}$ is a Lie algebra with a symmetric invariant bilinear form and from our proposition we have a surjective homomorphism $\psi$ : $\mathcal{G}(\hat{A}) \rightarrow \hat{\mathcal{L}}$ where $\hat{A}$ is an admissible indecomposable matrix. Moreover, using the same index $i_{0} \in I \subset J$ we used in the construction of $\psi$ we see that (2.16) and (2.17) now hold for the triple $(\mathcal{G}(\hat{A}), \hat{\mathcal{L}}, \psi)$ so all hypotheses of the Proposition 2.20 are satisfied and so we get a surjective homomorphism

$$
\hat{\psi}: \mathcal{G}(\hat{\hat{A}}) \rightarrow \hat{\hat{\mathcal{L}}}
$$

In other words, this process can be iterated. Indeed, we let aff $1(\mathcal{L})$ (respectively $\operatorname{aff}_{1}(A)$ ) equal $\hat{\mathcal{L}}$ (respectively $\hat{A}$ ) and inductively define $\operatorname{aff}_{n}(\mathcal{L})$ (respectively

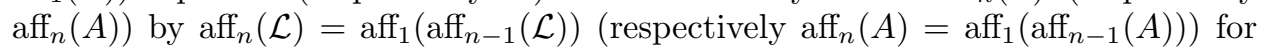
$n \geq 2$. Then we have a surjective homomorphism of $\mathcal{G}\left(\operatorname{aff}_{n}(A)\right)$ onto aff $n(\mathcal{L})$. Clearly, there is a surjective homomorphism of $\operatorname{aff}_{n}(\mathcal{L})$ onto $\mathcal{L} \otimes \mathbf{C}\left[t_{1}^{ \pm 1}, \ldots, t_{n}^{ \pm 1}\right]$, where $\mathbf{C}\left[t_{1}^{ \pm 1}, \ldots, t_{n}^{ \pm 1}\right]$ is the ring of finite Laurent polynomials in the commuting variables $t_{1}, \ldots, t_{n}$, so we then have a surjective homomorphism of $\mathcal{G}\left(\operatorname{aff}_{n}(A)\right)$ onto $\mathcal{L} \otimes \mathbf{C}\left[t_{1}^{ \pm 1}, \ldots, t_{n}^{ \pm 1}\right]$. 
We assume next that $A=\left(a_{i j}\right)_{i, j \in I}$ is symmetric and has even integer entries on the main diagonal. Let $\Gamma=\bigoplus_{i \in I} \mathbf{Z} \alpha_{i}$ be a lattice with basis $\left\{\alpha_{i}\right\}_{i \in I}$. We let the symmetric bilinear form $(\cdot, \cdot)$ on $\Gamma$ be defined by $\left(\alpha_{i}, \alpha_{j}\right)=a_{i j}$ for $i, j \in I$. We define a grading on the algebra $\mathcal{G}(A)$ by assigning $\operatorname{deg} e_{i}=\alpha_{i}, \operatorname{deg} f_{i}=-\alpha_{i}$, $\operatorname{deg} h_{i}=0$ for $i \in I$, then $\mathcal{G}(A)$ is a Lie algebra graded by the lattice $\Gamma$. We call an element $\alpha \in \Gamma$ a root if the set $\mathcal{G}^{\alpha}(A):=\{x \in \mathcal{G}(A) ; \operatorname{deg} x=\alpha\}$ is non-zero, and $\mathcal{G}^{\alpha}(A)$ is called the root space corresponding to a root $\alpha \in \Gamma$. Let $\mathcal{R}(A)$ be the ideal of $\mathcal{G}(A)$ generated by the root spaces $\mathcal{G}^{\alpha}(A)$ for which $(\alpha, \alpha)>2$. We now define the quotient Lie algebra

$$
\mathcal{L}(A):=\mathcal{G}(A) / \mathcal{R}(A)
$$

Note that if $A$ is a symmetric G.I.M. matrix, then $\mathcal{L}(A)$ is just a Slodowy I.M. algebra, and if $A$ is a Borcherds matrix, then $\mathcal{R}(A)=0$. In general, $\mathcal{R}(A)$ need not be zero (see $[\mathrm{BM}])$.

\section{VERTEX OPERATOR REPRESENTATION OF THE LIE ALGEBRAS}

In this section we first go towards defining the module $M(\Gamma)$ on which the moments of our vertex operators will act. Then we go on to establishing our generalization of the square length two cut off theorem of Goddard and Olive.

Let $\mathcal{X}$ be a Lie algebra. Let $\mathcal{Z}(\mathcal{X})$ be the center of $\mathcal{X}$ and $\mathcal{X}^{\prime}$ be the derived algebra of $\mathcal{X}$. We will call $\mathcal{X}$ a $\mathbf{Z}$-graded generalized Heisenberg Algebra if $\mathcal{X}=$ $\bigoplus_{n \in \mathbf{Z}} \mathcal{X}_{n}, \mathcal{X}_{0}=\mathcal{X}^{\prime}$ and

$$
\mathcal{X}^{\prime} \subseteq \mathcal{Z}(\mathcal{X}), \quad \operatorname{dim} \mathcal{X}^{\prime}=1
$$

Clearly, $\mathcal{X}$ contains two abelian subalgebras $\mathcal{X}^{+}=\bigoplus_{n \in \mathbf{Z}_{+}} \mathcal{X}_{n}, \mathcal{X}^{-}=\bigoplus_{n \in \mathbf{Z}_{+}} \mathcal{X}_{-n}$. Moreover, if we replace the condition $\mathcal{X}^{\prime} \subseteq \mathcal{Z}(\mathcal{X})$ by $\mathcal{X}^{\prime}=\mathcal{Z}(\mathcal{X})$ then $\mathcal{X}$ is the usual Z-graded Heisenberg algebra.

To study the vertex operator representation of the Lie algebra $\mathcal{G}(A)$ associated with the admissible matrix $A=\left(a_{i j}\right)_{i, j \in I}$ we will use stronger conditions on the matrix $A$ than in the previous section. Thus, from now on, we assume that the matrix $A$ satisfies the following conditions:

(C1) $a_{i j} \in \mathbf{Z}, a_{i i} \in 2 \mathbf{Z}$, and $a_{i i}>0$ implies $a_{i i}=2$, for $i, j \in I$.

(C2) $a_{i j}=a_{j i}$ for $i, j \in I$.

We form a symmetric bilinear form $(\cdot, \cdot)$ on a lattice $\Gamma=\bigoplus_{i \in I} \mathbf{Z} \alpha_{i}$ associated with the matrix $A$. That is, we define $(\cdot, \cdot): \Gamma \times \Gamma \rightarrow \mathbf{Z}$, such that $\left(\alpha_{i}, \alpha_{j}\right)=a_{i j}$ for $i, j \in I$. We then extend this form $\mathbf{C}$-bilinearly to the vector space $\mathcal{H}=\mathbf{C} \otimes_{\mathbf{Z}} \Gamma$. We view $\mathcal{H}$ as an abelian Lie algebra and, as usual, let $\mathcal{H}(n)$ be an isomorphic copy of $\mathcal{H}$ via the identification $a(n) \rightarrow a$ for $a \in \mathcal{H}, n \in \mathbf{Z}$. We form a $\mathbf{Z}$-graded Lie algebra

$$
\widetilde{\mathcal{H}}=\left(\bigoplus_{n \in \mathbf{Z}} \mathcal{H}(n)\right) \oplus \mathbf{C} \phi \oplus \mathbf{C} d,
$$

with the product

$$
\begin{gathered}
{[\alpha(m), \beta(n)]=m(\alpha, \beta) \delta_{m+n, 0} \phi} \\
{[d, \alpha(m)]=m \alpha(m), \quad[\phi, \widetilde{\mathcal{H}}]=0,}
\end{gathered}
$$

for $m, n \in \mathbf{Z}$ and $\alpha, \beta \in \mathcal{H}$.

Clearly, $\widetilde{\mathcal{H}}$ contains a generalized Heisenberg subalgebra $\widehat{\mathcal{H}}=\left(\bigoplus_{n \in \mathbf{Z} \backslash\{0\}} \mathcal{H}(n)\right)$ $\oplus \mathbf{C} \phi$, and if the bilinear form $(\cdot, \cdot)$ is non-degenerate on the vector space $\mathcal{H}$ then $\widehat{\mathcal{H}}$ is the usual Heisenberg algebra. 
Let $\mathcal{S}\left(\widehat{\mathcal{H}}^{-}\right)$be the symmetric algebra of $\widehat{\mathcal{H}}^{-}$, which is linearly spanned by monomials of the form $a_{1}\left(-n_{1}\right) \cdots a_{k}\left(-n_{k}\right)$ for $a_{1}, \ldots, a_{k} \in \mathcal{H}$ and $n_{1}, \ldots, n_{k} \in \mathbf{Z}_{+}$. Then $\mathcal{S}\left(\widehat{\mathcal{H}}^{-}\right)$affords a representation of the generalized Heisenberg algebra $\widehat{\mathcal{H}}$ by setting $\phi$ to act as $1, a(-m)$ to act as multiplication and $a(m)$ to act as the partial differential operator for which $a(m) \cdot b(-n)=m(a, b) \delta_{m-n, 0}$, where $m, n \in \mathbf{Z}_{+}$, $a, b \in \mathcal{H}$.

Let $\epsilon: \Gamma \times \Gamma \rightarrow\{1,-1\}$ be the 2-cocycle determined by

$$
\epsilon\left(\alpha_{i}, \alpha_{i}\right)=(-1)^{\left(\alpha_{i}, \alpha_{i}\right) / 2}, \quad \epsilon\left(\alpha_{i}, \alpha_{j}\right)= \begin{cases}1, & \text { if } i<j, \\ (-1)^{\left(\alpha_{i}, \alpha_{j}\right)}, & \text { if } i>j,\end{cases}
$$

and $\epsilon\left(\sum_{i} m_{i} \alpha_{i}, \sum_{j} n_{j} \alpha_{j}\right)=\prod_{i, j}\left(\epsilon\left(\alpha_{i}, \alpha_{j}\right)\right)^{m_{i} n_{j}}$, for $i, j \in I, \quad m_{i}, n_{j} \in \mathbf{Z}$. It is clear that $\epsilon$ satisfies the following conditions:

$$
\begin{gathered}
\epsilon(\alpha, \beta) \epsilon(\alpha+\beta, \gamma)=\epsilon(\beta, \gamma) \epsilon(\alpha, \beta+\gamma), \\
\epsilon(\alpha, \alpha)=(-1)^{(\alpha, \alpha) / 2}, \quad \epsilon(\alpha, \beta) \epsilon(\beta, \alpha)=(-1)^{(\alpha, \beta),}
\end{gathered}
$$

for $\alpha, \beta \in \Gamma$.

Let $\mathbf{C}[\Gamma]$ be the usual twisted group algebra of $\Gamma$ associated with the cocycle $\epsilon$. We denote by $e^{\alpha}$ for $\alpha \in \Gamma$ the base elements of $\mathbf{C}[\Gamma]$. The multiplication in $\mathbf{C}[\Gamma]$ is given by $e^{\alpha} e^{\beta}=\epsilon(\alpha, \beta) e^{\alpha+\beta}$, for $\alpha, \beta \in \Gamma$.

Set $V(\Gamma)=\mathbf{C}[\Gamma] \otimes \mathcal{S}\left(\widehat{\mathcal{H}}^{-}\right)$. For $h \in \mathcal{H}$, we define the operator $h(0)$ of $V(\Gamma)$ by $h(0) . e^{\alpha} \otimes u=(h, \alpha) e^{\alpha} \otimes u$, for $\alpha \in \Gamma, u \in \mathcal{S}\left(\widehat{\mathcal{H}}^{-}\right)$.

We remind the reader that for notation we are letting $\mathbf{N}, \mathbf{Z}, \mathbf{Z}_{+}, \mathbf{Z}_{-}$be the natural number, integers, positive integers, and negative integers respectively. Let $u, v, w, z, z_{1}, z_{2}, \ldots$ be formal variables. If $V$ is a vector space, we set

$$
V\{z\}=\left\{\sum_{n \in \mathbf{C}} v_{n} z^{n} \mid v_{n} \in V\right\}, \quad V\left[\left[z, z^{-1}\right]\right]=\left\{\sum_{n \in \mathbf{Z}} v_{n} z^{n} \mid v_{n} \in V\right\} .
$$

We also generalize these notations in the obvious way to the case of several commuting formal variables (see [FLM]). For $\alpha \in \Gamma$, we define

$$
E^{ \pm}(\alpha, z)=\exp \left(\sum_{n \in \pm \mathbf{Z}_{+}} \frac{\alpha(n)}{n} z^{-n}\right)
$$

and

$$
X(\alpha, z)=E^{-}(-\alpha, z) E^{+}(-\alpha, z) e^{\alpha} z^{\alpha+(\alpha, \alpha) / 2},
$$

where the operator $z^{\alpha+(\alpha, \alpha) / 2} \in(\operatorname{End} V(\Gamma))\{z\}$ is understood to be $z^{(\alpha, \alpha) / 2} z^{\alpha}$, and $z^{\alpha}$ is defined by $z^{\alpha} \cdot e^{\gamma} \otimes u=z^{(\alpha, \gamma)} e^{\gamma} \otimes u$, for $e^{\gamma} \otimes u \in V(\Gamma)$. It is clear that both $E^{ \pm}(\alpha, z)$ and $X(\alpha, z)$ can be expanded as formal power series in $z$ and $z^{-1}$ with coefficients (moment operators) acting on $V(\Gamma)$. It is easy to check the following identities (see $[\overline{F L M}])$ :

$$
\begin{aligned}
& {\left[h(m), E^{ \pm}(\alpha, z)\right]=-(h, \alpha) z^{m} E^{ \pm}(\alpha, z), \text { if } m \in \mp \mathbf{Z}_{+},} \\
& {\left[h(m), E^{ \pm}(\alpha, z)\right]=0, \text { if } m \in \pm \mathbf{Z}_{+} \text {or } m=0,}
\end{aligned}
$$

and

$$
\begin{gathered}
z^{\alpha} e^{\beta}=e^{\beta} z^{\alpha+(\alpha, \beta)}, \quad\left[h(m), e^{\alpha}\right]= \begin{cases}(h, \alpha) e^{\alpha}, & \text { if } m=0, \\
0, & \text { if } m \neq 0,\end{cases} \\
E^{+}(\alpha, z) E^{-}(\beta, w)=E^{-}(\beta, w) E^{+}(\alpha, z)\left(1-\frac{w}{z}\right)^{(\alpha, \beta)},
\end{gathered}
$$

for $\alpha, \beta \in \Gamma$. These identities give us 
Lemma 3.2. Let $\alpha, \beta \in \Gamma$, we have $[h(m), X(\alpha, z)]=(h, \alpha) z^{m} X(\alpha, z)$, for $m \in$ $\mathbf{Z}, h \in \mathcal{H}$, and

$$
\begin{gathered}
{[X(\alpha, z), X(\beta, w)]} \\
=z^{(\alpha, \alpha) / 2} w^{(\beta, \beta) / 2} E^{-}(-\alpha, z) E^{-}(-\beta, w) E^{+}(-\alpha, z) E^{+}(-\beta, w) e^{\alpha} e^{\beta} z^{\alpha} w^{\beta} P(z, w),
\end{gathered}
$$

where $P(z, w)=z^{(\alpha, \beta)}\left(1-\frac{w}{z}\right)^{(\alpha, \beta)}-(-1)^{(\alpha, \beta)} w^{(\alpha, \beta)}\left(1-\frac{z}{w}\right)^{(\alpha, \beta)}$.

Lemma 3.2 gives the following well known commutation relations (see [FLM] or EMY]:

Proposition 3.3. Let $\alpha, \beta \in \Gamma$. Then

$$
[X(\alpha, z), X(\beta, w)]= \begin{cases}0, & \text { if }(\alpha, \beta) \geq 0, \\ \epsilon(\alpha, \beta) \delta\left(\frac{w}{z}\right) X(\alpha+\beta, w), & \text { if }(\alpha, \beta)=-1, \\ -(D \delta)\left(\frac{w}{z}\right) \phi-\delta\left(\frac{w}{z}\right) \alpha(w), & \text { if } \alpha+\beta=0 \text { and }(\alpha, \alpha)=2,\end{cases}
$$

where $\alpha(w)=\sum_{n \in \mathbf{Z}} \alpha(n) w^{-n}$.

Let $J=\left\{i \in I \mid a_{i i} \leq 0\right\}$. Denote by $L=\bigoplus_{j \in J}\left(\mathbf{Z} \alpha_{j}^{+} \oplus \mathbf{Z} \alpha_{j}^{-}\right)$the free abelian group generated by $\left\{\alpha_{j}^{+}, \alpha_{j}^{-}\right\}_{j \in J}$. We form a symmetric bilinear form (also denoted by $(\cdot, \cdot))$ on $L$, by defining

$$
\left(\alpha_{i}^{+}, \alpha_{j}^{-}\right)=\delta_{i, j} \text { and }\left(\alpha_{i}^{+}, \alpha_{j}^{+}\right)=0=\left(\alpha_{i}^{-}, \alpha_{j}^{-}\right), i, j \in J .
$$

Let $L_{j}=\mathbf{Z} \alpha_{j}^{+} \oplus \mathbf{Z} \alpha_{j}^{-}$, for $j \in J$, and set $\mathcal{A}_{j}=L_{j} \otimes_{\mathbf{Z}} \mathbf{C}, \mathcal{A}=\bigoplus_{j \in J} \mathcal{A}_{j}$, and extend the symmetric form $(\cdot, \cdot) \mathbf{C}$-bilinearly to $\mathcal{A}$. Let $\Lambda=\Gamma \oplus L$, and $\Omega=\mathcal{H} \oplus \mathcal{A}$. We define a $\mathbf{C}$-bilinear symmetric form $(\cdot, \cdot): \Omega \times \Omega \rightarrow \mathbf{C}$ by requiring $(\mathcal{H}, \mathcal{A})=0$.

Our next goal is to define a generalized Heisenberg algebra associated to the even lattice $\Lambda$. For this purpose, we let $n \in-2 \mathbf{N}$, and let $\zeta_{n}: \mathbf{Z} \rightarrow \mathbf{C}$ be a function such that $\zeta_{n}(k)+\zeta_{n}(-k)=0$, for all $k \in \mathbf{Z}$. We define $\mathcal{Z}\left(\zeta_{n}\right):=\left\{k \in \mathbf{Z} \mid \zeta_{n}(k)=0\right\}$. Let $\mathcal{A}_{j}(m)$ be a copy of $\mathcal{A}_{j}$, for $j \in J, m \in \mathbf{Z}$. Then we form the Lie algebra $\widetilde{\Omega}$ associated to the lattice $\Lambda$ and the function $\zeta_{n}, n \in-2 \mathbf{N}$. That is

$$
\widetilde{\Omega}=\left(\bigoplus_{m \in \mathbf{Z}} \mathcal{H}(m)\right) \oplus\left(\bigoplus_{m \in \mathbf{Z}, j \in J} \mathcal{A}_{j}(m)\right) \oplus \mathbf{C} \phi
$$

with multiplication

$$
\begin{gathered}
{[\alpha(m), \beta(n)]=(\alpha, \beta) m \delta_{m+n, 0} \phi, \quad \text { if } \alpha \in \mathcal{H},} \\
{[\alpha(m), \beta(n)]=(\alpha, \beta) \zeta_{\alpha_{j}}(m) \delta_{m+n, 0} \phi, \quad \text { if } \alpha \in \mathcal{A}_{j}, \quad j \in J,} \\
{[\alpha(m), \phi]=0=[\phi, \alpha(m)],}
\end{gathered}
$$

where $\zeta_{\alpha_{j}}:=\zeta_{\left(\alpha_{j}, \alpha_{j}\right)}, \alpha, \beta \in \Omega$, and $m, n \in \mathbf{Z}$. It is clear that $\widetilde{\Omega}$ contains a generalized Heisenberg algebra $\widehat{\Omega}$ defined by

$$
\widehat{\Omega}=\left(\bigoplus_{m \in \mathbf{Z} \backslash\{0\}} \mathcal{H}(m)\right) \oplus\left(\bigoplus_{m \in \mathbf{Z} \backslash\{0\}} \mathcal{A}_{j}(m)\right) \oplus \mathbf{C} \phi .
$$

In what follows, we let $\zeta_{n}$ be defined by $\zeta_{n}(k)=\left(\begin{array}{c}k-\frac{n}{2} \\ 1-n\end{array}\right)$, for $n \in-2 \mathbf{N}$ and $k \in \mathbf{Z}$, where the symbol $\left(\begin{array}{l}a \\ k\end{array}\right)$ denotes the binomial coefficient

$$
\left(\begin{array}{l}
a \\
k
\end{array}\right)=\frac{a(a-1) \cdots(a-k+1)}{k !}
$$


for $a \in \mathbf{C}, k \in \mathbf{N}$. As usual, we take this to be 1 if $k=0$. It is clear that we have $\zeta_{n}(k)+\zeta_{n}(-k)=0$ and $\mathcal{Z}\left(\zeta_{n}\right)=\left\{i \in \mathbf{Z} ; \quad|i| \leq\left|\frac{n}{2}\right|\right\}$ for $n \in-2 \mathbf{N}$. For later use we record the following result.

Lemma 3.4. Let $a \in \mathbf{C}, n \in \mathbf{N}$. Then

$$
\sum_{i=0}^{n}(-1)^{n-i}\left(\begin{array}{c}
n \\
i
\end{array}\right)\left(\begin{array}{c}
a+i \\
n+j
\end{array}\right)=\left(\begin{array}{l}
a \\
j
\end{array}\right)
$$

for all $j \in \mathbf{N}$. In particular, we have $\sum_{i=0}^{n}(-1)^{n-i}\left(\begin{array}{c}n \\ i\end{array}\right)\left(\begin{array}{c}a+i \\ n+1\end{array}\right)=a$, for $n \in \mathbf{N}, a \in \mathbf{C}$.

Proof. Since

$$
(1+z)^{a} z^{n}=(1+z)^{a}(1+z-1)^{n}=\sum_{i=0}^{n}\left(\begin{array}{c}
n \\
i
\end{array}\right)(-1)^{n-i}(1+z)^{a+i},
$$

then, by using the formal binomial power series $(1+z)^{b}=\sum_{j=0}^{\infty}\left(\begin{array}{c}b \\ j\end{array}\right) z^{j}$, we immediately obtain (3.5) by comparing the coefficients of $z^{n+j}$ in (3.6).

As before, let $\mathcal{S}\left(\widehat{\Omega}^{-}\right)$be the symmetric algebra of $\widehat{\Omega}^{-}$, which is linearly spanned by monomials of the form $a_{1}\left(n_{1}\right) a_{2}\left(n_{2}\right) \cdots a_{k}\left(n_{k}\right)$, for $a_{i} \in\left(\bigcup_{j \in J} \mathcal{A}_{j}\right) \cup \mathcal{H}, n_{i} \in$ $-\mathbf{Z}_{+}$. Then $\mathcal{S}\left(\widehat{\Omega}^{-}\right)$gives a representation of the generalized Heisenberg algebra $\widehat{\Omega}$ by setting $\phi$ to act as $1, a(-m)$ to act as multiplication, and $b(n)$ to act as the partial differential operator, for which

$$
b(n) . a(-m)= \begin{cases}(b, a) n \delta_{n-m, 0} & \text { if } b \in \mathcal{H}, \\ (b, a) \zeta_{\alpha_{j}}(n) \delta_{n-m, 0} & \text { if } b \in \mathcal{A}_{j}, \text { for some } j \in J,\end{cases}
$$

where $n, m \in \mathbf{Z}_{+}$and $a, b \in\left(\bigcup_{j \in J} \mathcal{A}_{j}\right) \cup \mathcal{H}$. Moreover, we extend $\mathcal{S}\left(\widehat{\Omega}^{-}\right)$to be an $\widetilde{\Omega}$-module by requiring $a(0)$ to act trivially on $\mathcal{S}\left(\widehat{\Omega}^{-}\right)$for $a \in\left(\bigcup_{j \in J} \mathcal{A}_{j}\right) \cup \mathcal{H}$. Clearly, if $k \in \mathcal{Z}\left(\zeta_{j}\right)$ for some $j \in J$, then $a(k)$ acts trivially on $\mathcal{S}\left(\widehat{\Omega}^{-}\right)$for all $a \in \mathcal{A}_{j}$.

Now we form a vector space

$$
M(\Gamma)=\mathbf{C}[\Gamma] \otimes \mathcal{S}\left(\widehat{\Omega}^{-}\right) .
$$

This is the full Fock space on which we will define the vertex operators. It is clear that $M(\Gamma)$ contains $V(\Gamma)$ as a subspace, and as vector spaces we have $\mathcal{S}\left(\widehat{\Omega}^{-}\right) \simeq$ $\mathcal{S}\left(\widehat{\mathcal{H}}^{-}\right) \otimes \mathcal{S}\left(\widehat{\mathcal{A}}^{-}\right)$, where $\widehat{\mathcal{A}}=\bigoplus_{m \in \mathbf{Z} \backslash\{0\}}\left(\mathbf{C} \alpha_{j}^{+}(m) \oplus \mathbf{C} \alpha_{j}^{-}(m)\right)$.

For simplicity, we set $\phi(n)=\delta_{n, 0} \phi$ for $n \in \mathbf{Z}$, and then for $\beta \in L \oplus \mathbf{Z} \phi$ we define $\beta(z)=\sum_{n \in \mathbf{Z}} \beta(n) z^{-n}$. We shall use normal ordering symbols, indicated by colons, which indicate that the enclosed operators are to be reordered so that all the operators $\alpha(n)\left(\alpha \in \Gamma \cup\{L \oplus \mathbf{Z} \phi\}, n \in-\mathbf{Z}_{+}\right)$are to be placed to the left of all operators $\alpha(n)$ and $z^{\beta}\left(\alpha \in \Gamma \cup\{L \oplus \mathbf{Z} \phi\}, \beta \in \Gamma, n \in \mathbf{Z}_{+}\right)$before the expression is applied. We now define our vertex operators, for $\alpha \in \Gamma, \beta \in L \oplus \mathbf{Z} \phi$,

$$
X^{(\beta)}(\alpha, z)=: X(\alpha, z) \beta(z):=E^{-}(-\alpha, z) \beta(z) E^{+}(-\alpha, z) e^{\alpha} z^{\alpha+\frac{1}{2}(\alpha, \alpha)},
$$

where the operators $e^{\alpha}$ and $z^{\alpha}$ are understood to act as $e^{\alpha} \otimes 1$ and $z^{\alpha} \otimes 1$ respectively on $M(\Gamma)$. 
One may express the vertex operator $X^{(\beta)}(\alpha, z)$ as a formal power series in $z$, that is

$$
X^{(\beta)}(\alpha, z)=\sum_{n \in \mathbf{Z}} x_{n}^{(\beta)}(\alpha) z^{-n}
$$

where the moments $x_{n}^{(\beta)}(\alpha), n \in \mathbf{Z}$ are operators on $M(\Gamma)$. Note that $\phi(z)=$ $\sum_{n \in \mathbf{Z}} \phi(n) z^{-n}=1$ and $X^{(\beta)}(\gamma, z)=: X(\gamma, z) \beta(z)$, then we have $X^{\phi}(\gamma, z)=X(\gamma, z)$. Therefore we have

Proposition 3.9 ([EMY $])$. The operators $x_{n}^{(\not)}\left(\alpha_{i}\right)$ are locally nilpotent on $M(\Gamma)$ for all $n \in \mathbf{Z}$, and $i \in I \backslash J$.

We will use the following Lemma. Its proof can be found in [FLM].

Lemma 3.10. Let $V$ be a vector space, $a \in \mathbf{C}^{\times}$, and $Y(z, w) \in(E n d V)\left[\left[z, z^{-1}\right.\right.$, $\left.\left.w, w^{-1}\right]\right]$, such that $\lim _{w \rightarrow z} Y(z, w)$ exists (that is, if $Y(z, w)$ is applied to any element of $V$, and set $w=z$, the coefficient of any monomial in $z$ is a finite sum in $V$; see [FLM] $)$. Then, for $k \in \mathbf{N}$

$$
Y(z, w) \partial_{w}^{k} \delta\left(a \frac{w}{z}\right)=\sum_{l=0}^{k}(-1)^{l}\left(\begin{array}{c}
k \\
l
\end{array}\right)\left(\partial_{w}^{l} Y\right)\left(z, a^{-1} z\right) \partial_{w}^{k-l} \delta\left(a \frac{w}{z}\right),
$$

with all expressions existing. In particular,

$$
\begin{gathered}
Y(z, w)(D \delta)\left(a \frac{w}{z}\right)=Y\left(z, a^{-1} z\right)(D \delta)\left(a \frac{w}{z}\right)-\left(D_{w} Y\right)\left(z, a^{-1} z\right) \delta\left(a \frac{w}{z}\right) \\
Y(z, w) \delta\left(a \frac{w}{z}\right)=Y\left(z, a^{-1} z\right) \delta\left(a \frac{w}{z}\right)
\end{gathered}
$$

where $D_{w}=w \partial_{w}, \partial_{w}^{k}=\left(\frac{\partial}{\partial w}\right)^{k}$.

We now begin our analysis of the power series which enter in when we compute commutators of the vertex operators. For $m \in \mathbf{Z}$, we define power series

$$
f_{m}(z, w)=z^{-m}\left(1-\frac{w}{z}\right)^{-m}, \quad g_{m}(z, w)=(-w)^{-m}\left(1-\frac{z}{w}\right)^{-m} .
$$

As usual, these are just shorthand for the associated power series expansions. The reader should recall that $(1+z)^{a}=\sum_{j=0}^{\infty}\left(\begin{array}{c}a \\ j\end{array}\right) z^{j}$. One can easily check the following facts:

$$
f_{-m}(z, w)=g_{-m}(z, w)=(z-w)^{m}
$$

for $m \in \mathbf{N}$, and also

$$
f_{m}(z, w) f_{n}(z, w)=f_{m+n}(z, w), \quad g_{m}(z, w) g_{n}(z, w)=g_{m+n}(z, w),
$$

for $m, n \in \mathbf{Z}$. Moreover, we have

Lemma 3.15. Let $m \in \mathbf{Z}$, then

$$
\begin{gathered}
f_{m}(z, w) f_{-m}(z, w)=g_{m}(z, w) g_{-m}(z, w)=g_{m}(z, w) f_{-m}(z, w)=1, \\
g_{m}(z, w) f_{-m}(w, z)=(-1)^{m} \\
f_{m}(z, w) f_{-m}(w, z)=g_{m}(z, w) g_{-m}(w, z)=(-1)^{m} .
\end{gathered}
$$


Proof. (3.16) follows from (3.13) and (3.14). For (3.17), if $m \geq 0$ we have

$$
\begin{gathered}
g_{m}(z, w) f_{-m}(w, z)=g_{m}(z, w)(w-z)^{m}=(-1)^{m} g_{m}(z, w)(z-w)^{m} \\
=(-1)^{m} g_{m}(z, w) g_{-m}(z, w)=(-1)^{m},
\end{gathered}
$$

as required. Similarly, for the case $m<0$. Finally, (3.18) follows from (3.13) and (3.17).

Lemma 3.19. Let $m \in \mathbf{Z}$. We have

$$
\begin{aligned}
& \partial_{w} f_{m}(z, w)=m f_{m+1}(z, w), \quad \partial_{z} f_{m}(z, w)=-m f_{m+1}(z, w), \\
& \partial_{w} g_{m}(z, w)=m g_{m+1}(z, w), \quad \partial_{z} g_{m}(z, w)=-m g_{m+1}(z, w),
\end{aligned}
$$

Proof. Note that $f_{m}(z, w)=z^{-m}\left(1-\frac{w}{z}\right)^{-m}=z^{-m} \sum_{k=0}^{\infty}\left(\begin{array}{c}-m \\ k\end{array}\right)\left(-\frac{w}{z}\right)^{k}$, so we have

$$
\begin{gathered}
\partial_{w} f_{m}(z, w)=z^{-m} \sum_{k=1}^{\infty} k\left(\begin{array}{c}
-m \\
k
\end{array}\right)\left(-\frac{w}{z}\right)^{k-1}\left(-z^{-1}\right) \\
=-z^{-m-1} \sum_{k=1}^{\infty} k\left(\begin{array}{c}
-m \\
k
\end{array}\right)\left(-\frac{w}{z}\right)^{k-1} .
\end{gathered}
$$

Since $k\left(\begin{array}{c}-m \\ k\end{array}\right)=-m\left(\begin{array}{c}-m-1 \\ k-1\end{array}\right)$, we obtain

$$
\begin{gathered}
\partial_{w} f_{m}(z, w)=-z^{-m-1} \sum_{k=1}^{\infty}(-m)\left(\begin{array}{c}
-m-1 \\
k-1
\end{array}\right)\left(-\frac{w}{z}\right)^{k-1} \\
=m z^{-m-1}\left(1-\frac{w}{z}\right)^{-m-1}=m f_{m+1}(z, w) .
\end{gathered}
$$

This proves the first identity. For the second identity we have

$$
\begin{aligned}
\partial_{z} f_{m}(z, w) & =-m z^{-m-1} \sum_{k=0}^{\infty}\left(\begin{array}{c}
-m \\
k
\end{array}\right)\left(-\frac{w}{z}\right)^{k}-z^{-m} \sum_{k=1}^{\infty} k\left(\begin{array}{c}
-m \\
k
\end{array}\right)\left(-\frac{w}{z}\right)^{k} z^{-1} \\
& =-m z^{-m-1}\left(1+\sum_{k=1}^{\infty}\left\{\left(\begin{array}{c}
-m \\
k
\end{array}\right)-\left(\begin{array}{c}
-m-1 \\
k-1
\end{array}\right)\right\}\left(-\frac{w}{z}\right)^{k}\right) .
\end{aligned}
$$

Moreover, since $\left(\begin{array}{c}-m \\ k\end{array}\right)-\left(\begin{array}{c}-m-1 \\ k-1\end{array}\right)=\left(\begin{array}{c}-m-1 \\ k\end{array}\right)$ for $k \geq 1$, we have

$$
\begin{gathered}
\partial_{z} f_{m}(z, w)=-m z^{-m-1}\left(1+\sum_{k=1}^{\infty}\left(\begin{array}{c}
-m-1 \\
k
\end{array}\right)\left(-\frac{w}{z}\right)^{k}\right) \\
=-m z^{-m-1}\left(1-\frac{w}{z}\right)^{-m-1}=-m f_{m+1}(z, w),
\end{gathered}
$$

as required. The other cases can be proved similarly.

Lemma 3.20. Let $m \in \mathbf{Z}$. Then

$$
f_{m}(z, w)-g_{m}(z, w)= \begin{cases}0, & \text { if } m \leq 0 \\ w^{-1} \frac{(-1)^{m-1}}{(m-1) !} \partial_{z}^{m-1} \delta\left(\frac{z}{w}\right), & \text { if } m \geq 1\end{cases}
$$


Proof. If $m \leq 0$, the result follows from (3.13). Now suppose $m \geq 1$, we want to prove the result by induction on $m \geq 1$. First, we have

$$
\begin{aligned}
& f_{1}(z, w)-g_{1}(z, w)=z^{-1}\left(1-\frac{w}{z}\right)^{-1}-(-w)^{-1}\left(1-\frac{z}{w}\right)^{-1} \\
= & z^{-1} \sum_{k=0}^{\infty}\left(\frac{w}{z}\right)^{k}+w^{-1} \sum_{k=0}^{\infty}\left(\frac{z}{w}\right)^{k}=w^{-1} \sum_{k \in \mathbf{Z}}\left(\frac{z}{w}\right)^{k}=w^{-1} \delta\left(\frac{z}{w}\right) .
\end{aligned}
$$

Finally, by induction and Lemma 3.19

$$
\begin{aligned}
& f_{m+1}(z, w)-g_{m+1}(z, w)=-\frac{1}{m} \partial_{z}\left(f_{m}(z, w)-g_{m}(z, w)\right) \\
= & -\frac{1}{m} \partial_{z}\left(w^{-1} \frac{(-1)^{m-1}}{(m-1) !} \partial_{z}^{m-1} \delta\left(\frac{z}{w}\right)\right)=w^{-1} \frac{(-1)^{m}}{m !} \partial_{z}^{m} \delta\left(\frac{z}{w}\right),
\end{aligned}
$$

as required.

Theorem 3.22. Let $k \geq 2, \lambda_{i j} \in \mathbf{Z}$, and $i, j=1,2, \ldots, k$ be such that

$$
\sum_{1 \leq i<j \leq k} \lambda_{i j}<k-1
$$

Then

$$
\prod_{l=2}^{k}\left(\prod_{i=1}^{l-1} f_{\lambda_{i l}}\left(z_{i}, z_{l}\right)-\prod_{i=1}^{l-1} g_{\lambda_{i l}}\left(z_{i}, z_{l}\right)\right)=0
$$

Proof. When $k=2$, (3.23) follows from Lemma 3.20. We now suppose $k \geq 3$, and rewrite the left hand side of (3.23) as follows:

$$
\left(f_{\lambda_{12}}\left(z_{1}, z_{2}\right)-g_{\lambda_{12}}\left(z_{1}, z_{2}\right)\right)\left\{\prod_{l=3}^{k}\left(\prod_{i=1}^{l-1} f_{\lambda_{i l}}\left(z_{i}, z_{l}\right)-\prod_{i=1}^{l-1} g_{\lambda_{i l}}\left(z_{i}, z_{l}\right)\right)\right\}
$$

Therefore, we only need to consider the case for $\lambda_{12} \geq 1$.

Put $f\left(z_{1}, \ldots, z_{k}\right)=\prod_{l=3}^{k}\left(\prod_{i=1}^{l-1} f_{\lambda_{i l}}\left(z_{i}, z_{l}\right)-\prod_{i=1}^{l-1} g_{\lambda_{i l}}\left(z_{i}, z_{l}\right)\right)$. By Lemma 3.20, we have

$$
\begin{aligned}
& \left(f_{\lambda_{12}}\left(z_{1}, z_{2}\right)-g_{\lambda_{12}}\left(z_{1}, z_{2}\right)\right) f\left(z_{1}, \ldots, z_{k}\right)=z_{2}^{-1} \frac{(-1)^{\lambda_{12}-1}}{\left(\lambda_{12}-1\right) !} f\left(z_{1}, \ldots, z_{k}\right) \partial_{z_{1}}^{\lambda_{12}-1} \delta\left(\frac{z_{1}}{z_{2}}\right) \\
& \quad=z_{2}^{-1} \frac{(-1)^{\lambda_{12}-1}}{\left(\lambda_{12}-1\right) !} \sum_{j=0}^{\lambda_{12}-1}(-1)^{j}\left(\begin{array}{c}
\lambda_{12}-1 \\
j
\end{array}\right)\left(\partial_{z_{1}}^{j} f\right)\left(z_{2}, z_{2}, z_{3}, \ldots, z_{k}\right) \partial_{z_{1}}^{\lambda_{12}-1-j} \delta\left(\frac{z_{1}}{z_{2}}\right)
\end{aligned}
$$

where we have used Lemma 3.10. To prove (3.23), we are going to show $\left(\partial_{z_{1}}^{j} f\right)\left(z_{2}, z_{2}, z_{3}, \ldots, z_{k}\right)=0$ for $0 \leq j \leq \lambda_{12}-1$. By Lemma 3.19, we have $\frac{\partial^{j} f}{\partial z_{1}^{j}}\left(z_{1}, \ldots, z_{k}\right)=$ a linear combination of terms of the form

$$
\prod_{l=3}^{k}\left(\prod_{i=1}^{l-1} f_{\lambda_{i l}+n_{i l}}\left(z_{i}, z_{l}\right)-\prod_{i=1}^{l-1} g_{\lambda_{i l}+n_{i l}}\left(z_{i}, z_{l}\right)\right)
$$


where $n_{i l} \in \mathbf{N}$ such that $n_{i l}=0$ for $i \geq 2$, and $\sum_{l=3}^{k} n_{1 l}=j$. Thus, by (3.14) we substitute $z_{1}$ to be $z_{2}$ in the above and obtain $\frac{\partial^{j} f}{\partial z_{1}^{j}}\left(z_{2}, z_{2}, z_{3}, \ldots, z_{k}\right)=$ a linear combination of terms of the form

$$
\prod_{l=3}^{k}\left(\prod_{i=2}^{l-1} f_{\bar{\lambda}_{i l}}\left(z_{i}, z_{l}\right)-\prod_{i=2}^{l-1} g_{\bar{\lambda}_{i l}}\left(z_{i}, z_{l}\right)\right)
$$

where

$$
\bar{\lambda}_{i l}= \begin{cases}\lambda_{1 l}+n_{1 l}+\lambda_{2 l}, & \text { if } i=2 \\ \lambda_{i l} & \text { if } i \geq 3\end{cases}
$$

We may rewrite (3.25) in the following form:

$$
\prod_{l=2}^{k-1}\left(\prod_{i=1}^{l-1} f_{\bar{\lambda}_{i+1, l+1}}\left(z_{i+1}, z_{l+1}\right)-\prod_{i=1}^{l-1} g_{\bar{\lambda}_{i+1, l+1}}\left(z_{i+1}, z_{l+1}\right)\right)
$$

and we claim this is zero by induction using the $(k-1)$-case. To see this, we only need to check the inequality $\sum_{1 \leq i<j \leq k-1} \bar{\lambda}_{i+1, j+1}<k-2$. Indeed,

$$
\begin{aligned}
& \sum_{1 \leq i<j \leq k-1} \bar{\lambda}_{i+1, j+1}=\sum_{2 \leq i<j \leq k} \bar{\lambda}_{i j}=\sum_{2<j \leq k}\left(\lambda_{1 j}+n_{1 j}+\lambda_{2 j}\right)+\sum_{3 \leq i<j \leq k} \lambda_{i j} \\
\leq & \sum_{2<j \leq k}\left(\lambda_{1 j}+\lambda_{2 j}\right)+\left(\sum_{3 \leq i<j \leq k} \lambda_{i j}\right)+\left(\lambda_{12}-1\right)=\left(\sum_{1 \leq i<j \leq k} \lambda_{i j}\right)-1<k-2,
\end{aligned}
$$

as required.

Lemma 3.26. Let $m \in \mathbf{Z}$. Then

$$
g_{1-m}(z, w)\left(f_{m}(z, w)-g_{m}(z, w)\right)= \begin{cases}0, & \text { if } m \leq 0 \\ w^{-1} \delta\left(\frac{z}{w}\right), & \text { if } m \geq 1\end{cases}
$$

Proof. We only need to consider the case for $m \geq 1$. By (3.13) and (3.14), we have

$$
\begin{gathered}
g_{1-m}(z, w)\left(f_{m}(z, w)-g_{m}(z, w)\right)=g_{1-m}(z, w) f_{m}(z, w)-g_{1-m}(z, w) g_{m}(z, w) \\
=f_{1-m}(z, w) f_{m}(z, w)-g_{1-m}(z, w) g_{m}(z, w)=f_{1}(z, w)-g_{1}(z, w) .
\end{gathered}
$$

This gives the result by using Lemma 3.20 .

Corollary 3.27. Let $m \in \mathbf{Z}$. Then

$$
\left.\left(D_{u} g_{-m}(z, u)\right)\right|_{u=w}\left(f_{m}(z, w)-g_{m}(z, w)\right)=-\frac{m+|m|}{2} \delta\left(\frac{z}{w}\right) .
$$

Proof. Applying Lemma 3.19, we have

$$
D_{u} g_{-m}(z, u)=u \partial_{u} g_{-m}(z, u)=-m u g_{1-m}(z, u) .
$$

Thus the result follows from this and Lemma 3.26.

Lemma 3.28. Let $\lambda_{i}, \lambda_{i}^{\prime}(i \geq 1)$ be integers satisfying $\lambda_{i}+\lambda_{i}^{\prime}=0$. Then for $n>1$

$$
\prod_{\tau=1}^{n-1} g_{\lambda_{\tau}}\left(z_{\tau}, z_{n}\right)\left(\prod_{\tau=1}^{n-1} f_{\lambda_{\tau}^{\prime}}\left(z_{\tau}, z_{n}\right)-\prod_{\tau=1}^{n-1} g_{\lambda_{\tau}^{\prime}}\left(z_{\tau}, z_{n}\right)\right)=0 .
$$

Proof. This follows from Lemma 3.15. 
Lemma 3.30. Under the conditions of Lemma 3.28, we have

$$
\begin{gathered}
\left.D_{z_{m}}\left(\prod_{\tau=1}^{n-1} g_{\lambda_{\tau m}}\left(z_{\tau}, z_{m}\right)\right)\right|_{z_{m}=z_{n}}\left(\prod_{\tau=1}^{n-1} f_{\lambda_{\tau n}}\left(z_{\tau}, z_{n}\right)-\prod_{\tau=1}^{n-1} g_{\lambda_{\tau n}}\left(z_{\tau}, z_{n}\right)\right) \\
=-\sum_{\tau=1}^{n-1} \frac{\lambda_{\tau n}+\left|\lambda_{\tau n}\right|}{2} \delta\left(z_{\tau} / z_{n}\right)
\end{gathered}
$$

Proof. Note that

$$
\left.D_{z_{m}}\left(\prod_{\tau=1}^{n-1} g_{\lambda_{\tau m}}\left(z_{\tau}, z_{m}\right)\right)\right|_{z_{m}=z_{n}}=\left.\sum_{p=1}^{n-1}\left(\prod_{\substack{\tau=1 \\ \tau \neq p}}^{n-1} g_{\lambda_{\tau m}}\left(z_{\tau}, z_{n}\right)\right) D_{z_{m}}\left(g_{\lambda_{p m}}\left(z_{p}, z_{m}\right)\right)\right|_{z_{m}=z_{n}}
$$

so we obtain, by Lemma 3.15 and Corollary 3.27

$$
\begin{gathered}
\left.D_{z_{m}}\left(\prod_{\tau=1}^{n-1} g_{\lambda_{\tau m}}\left(z_{\tau}, z_{m}\right)\right)\right|_{z_{m}=z_{n}}\left(\prod_{\tau=1}^{n-1} f_{\lambda_{\tau n}}\left(z_{\tau}, z_{n}\right)-\prod_{\tau=1}^{n-1} g_{\lambda_{\tau n}}\left(z_{\tau}, z_{n}\right)\right) \\
=\left.\sum_{p=1}^{n-1} D_{z_{m}}\left(g_{\lambda_{p m}}\left(z_{p}, z_{m}\right)\right)\right|_{z_{m}=z_{n}}\left(f_{\lambda_{p n}}\left(z_{p}, z_{n}\right)-g_{\lambda_{p n}}\left(z_{p}, z_{n}\right)\right) \\
=\sum_{p=1}^{n-1}\left(-\frac{\lambda_{p n}+\left|\lambda_{p n}\right|}{2} \delta\left(z_{p} / z_{n}\right)\right),
\end{gathered}
$$

as required.

Let $\gamma_{1}, \ldots, \gamma_{k} \in \Gamma, k \geq 2$. For simplicity, we define the following power series:

$$
\begin{gathered}
F_{j}=F_{j}\left(z_{1}, \cdots, z_{j}\right)= \begin{cases}1, & \text { if } j=1, \\
\prod_{i=1}^{j-1} f_{-\left(\gamma_{i}, \gamma_{j}\right)}\left(z_{i}, z_{j}\right)-\prod_{i=1}^{j-1} g_{-\left(\gamma_{i}, \gamma_{j}\right)}\left(z_{i}, z_{j}\right), & \text { if } j>1,\end{cases} \\
G_{j}=G_{j}\left(z_{1}, \cdots, z_{j}\right)= \begin{cases}1, & \text { if } j=1, \\
\prod_{i=1}^{j-1} g_{-\left(\gamma_{i}, \gamma_{j}\right)}\left(z_{i}, z_{j}\right), & \text { if } j>1 .\end{cases}
\end{gathered}
$$

For $x_{1}, \ldots, x_{n}$ elements of a Lie algebra, $\left[x_{1}, \cdots, x_{n}\right]$ means $\left(\operatorname{ad} x_{1}\right) \cdots\left(\operatorname{ad} x_{n-1}\right) x_{n}$ for $n \geq 2$.

Proposition 3.32. Let $\gamma_{1}, \ldots, \gamma_{k} \in \Gamma, \beta_{1}, \ldots, \beta_{k} \in L \oplus \mathbf{Z} \not, k \geq 2$. Then

$$
\begin{gathered}
{\left[X^{\left(\beta_{k}\right)}\left(\gamma_{k}, z_{k}\right), \cdots, X^{\left(\beta_{1}\right)}\left(\gamma_{1}, z_{1}\right)\right]=(-1)^{k-1}\left(\prod_{i=1}^{k} z_{i}^{\left(\gamma_{i}, \gamma_{i}\right) / 2}\right)} \\
\cdot\left(\prod_{i=1}^{k} E^{-}\left(-\gamma_{i}, z_{i}\right)\right) Q\left(z_{1}, \cdots, z_{k}, \beta_{1}, \cdots, \beta_{k}\right)\left(\prod_{i=1}^{k} E^{+}\left(-\gamma_{i}, z_{i}\right)\right)\left(e^{\gamma_{1}} \cdots e^{\gamma_{k}}\right)\left(\prod_{i=1}^{k} z_{i}^{\gamma_{i}}\right),
\end{gathered}
$$

where $Q\left(z_{1}, \cdots, z_{k}, \beta_{1}, \cdots, \beta_{k}\right)=$ a linear combination of terms of the form

$$
\prod_{m=1}^{l}\left(\left[\beta_{i_{m}}\left(z_{i_{m}}\right), \beta_{j_{m}}\left(z_{j_{m}}\right)\right] F_{i_{m}} G_{j_{m}}\right)\left(\prod_{n=1}^{s} F_{t_{n}}\right)\left(\beta_{t_{1}}\left(z_{t_{1}}\right) \cdots \beta_{t_{s}}\left(z_{t_{s}}\right)\right),
$$


in which $l, s \geq 0,\left(i_{1}, \ldots, i_{l}, j_{1}, \ldots, j_{l}, t_{1}, \ldots, t_{s}\right)$ is some permutation of $\{1,2, \ldots, k\}$, such that

1) $i_{m}<j_{m}$, for $1 \leq m \leq l$,

2) $j_{1}<j_{2}<\cdots<j_{l}, \quad t_{1}<t_{2}<\cdots<t_{s}$.

Remark 3.33. If $\left(\beta_{i}, \beta_{j}\right)=0$ for all $1 \leq i \neq j \leq k$, then

$$
Q\left(z_{1}, \ldots, z_{k}, \beta_{1}, \ldots, \beta_{k}\right)=\left(\prod_{j=1}^{k} F_{j}\right)\left(\beta_{1}\left(z_{1}\right) \ldots \beta_{k}\left(z_{k}\right)\right),
$$

since $\left[\beta_{i}\left(z_{i}\right), \beta_{j}\left(z_{j}\right)\right]=0$ if $\left(\beta_{i}, \beta_{j}\right)=0$.

Proof of Proposition 3.32. Since

$$
E^{+}\left(\gamma_{i}, z_{i}\right) E^{-}\left(\gamma_{j}, z_{j}\right)=E^{-}\left(\gamma_{j}, z_{j}\right) E^{+}\left(\gamma_{i}, z_{i}\right)\left(1-\frac{z_{j}}{z_{i}}\right)^{\left(\gamma_{i}, \gamma_{j}\right)},
$$

$$
z_{i}^{\gamma_{i}} e^{\gamma_{j}}=e^{\gamma_{j}} z_{i}^{\gamma_{i}+\left(\gamma_{i}, \gamma_{j}\right)}, \quad e^{\gamma_{i}} e^{\gamma_{j}}=\epsilon\left(\gamma_{i}, \gamma_{j}\right) e^{\gamma_{i}+\gamma_{j}}=(-1)^{\left(\gamma_{i}, \gamma_{j}\right)} e^{\gamma_{j}} e^{\gamma_{i}},
$$

and

and

$$
\begin{gathered}
E^{ \pm}\left(\gamma_{i}, z_{i}\right) \beta_{j}\left(z_{j}\right)=\beta_{j}\left(z_{j}\right) E^{ \pm}\left(\gamma_{i}, z_{i}\right), \\
z_{i}^{\gamma_{i}} \beta_{j}\left(z_{j}\right)=\beta_{j}\left(z_{j}\right) z_{i}^{\gamma_{i}}, \quad e^{\gamma_{i}} \beta_{j}\left(z_{j}\right)=\beta_{j}\left(z_{j}\right) e^{\gamma_{i}},
\end{gathered}
$$

for $i \neq j$, we have

$$
\begin{gathered}
X^{\left(\beta_{2}\right)}\left(\gamma_{2}, z_{2}\right) X^{\left(\beta_{1}\right)}\left(\gamma_{1}, z_{1}\right)=E^{-}\left(-\gamma_{2}, z_{2}\right) \beta_{2}\left(z_{2}\right) E^{+}\left(-\gamma_{2}, z_{2}\right) e^{\gamma_{2}} z_{2}^{\gamma_{2}+\left(\gamma_{2}, \gamma_{2}\right) / 2} \\
\cdot E^{-}\left(-\gamma_{1}, z_{1}\right) \beta_{1}\left(z_{1}\right) E^{+}\left(-\gamma_{1}, z_{1}\right) e^{\gamma_{1}} z_{1}^{\gamma_{1}+\left(\gamma_{1}, \gamma_{1}\right) / 2} \\
=E^{-}\left(-\gamma_{2}, z_{2}\right) E^{-}\left(-\gamma_{1}, z_{1}\right) \beta_{2}\left(z_{2}\right) \beta_{1}\left(z_{1}\right) E^{+}\left(-\gamma_{2}, z_{2}\right) E^{+}\left(-\gamma_{1}, z_{1}\right) e^{\gamma_{2}} z_{2}^{\gamma_{2}+\left(\gamma_{2}, \gamma_{2}\right) / 2} \\
\cdot e^{\gamma_{1}} z_{1}^{\gamma_{1}+\left(\gamma_{1}, \gamma_{1}\right) / 2}\left(1-\frac{z_{1}}{z_{2}}\right)^{\left(\gamma_{1}, \gamma_{2}\right)} \\
=z_{1}^{\left(\gamma_{1}, \gamma_{1}\right) / 2} z_{2}^{\left(\gamma_{2}, \gamma_{2}\right) / 2} E^{-}\left(-\gamma_{2}, z_{2}\right) E^{-}\left(-\gamma_{1}, z_{1}\right)\left(-\left[\beta_{1}\left(z_{1}\right), \beta_{2}\left(z_{2}\right)\right]+\beta_{1}\left(z_{1}\right) \beta_{2}\left(z_{2}\right)\right) \\
\cdot E^{+}\left(-\gamma_{2}, z_{2}\right) E^{+}\left(-\gamma_{1}, z_{1}\right) e^{\gamma_{2}} e^{\gamma_{1}} z_{2}^{\gamma_{2}} z_{1}^{\gamma_{1}} z_{2}^{\left(\gamma_{1}, \gamma_{2}\right)}\left(1-\frac{z_{1}}{z_{2}}\right)^{\left(\gamma_{1}, \gamma_{2}\right)} \\
=z_{1}^{\left(\gamma_{1}, \gamma_{1}\right) / 2} z_{2}^{\left(\gamma_{2}, \gamma_{2}\right) / 2} E^{-}\left(-\gamma_{2}, z_{2}\right) E^{-}\left(-\gamma_{1}, z_{1}\right)\left(-\left[\beta_{1}\left(z_{1}\right), \beta_{2}\left(z_{2}\right)\right]+\beta_{1}\left(z_{1}\right) \beta_{2}\left(z_{2}\right)\right) \\
\cdot E^{+}\left(-\gamma_{2}, z_{2}\right) E^{+}\left(-\gamma_{1}, z_{1}\right) e^{\gamma_{1}} e^{\gamma_{2}} z_{2}^{\gamma_{2}} z_{1}^{\gamma_{1}} z_{2}^{\left(\gamma_{1}, \gamma_{2}\right)}\left(1-\frac{z_{1}}{z_{2}}\right)\left(\gamma_{1}, \gamma_{2}\right)(-1)^{\left(\gamma_{1}, \gamma_{2}\right)} \\
=z_{1}^{\left(\gamma_{1}, \gamma_{1}\right) / 2} z_{2}^{\left(\gamma_{2}, \gamma_{2}\right) / 2} E^{-}\left(-\gamma_{1}, z_{1}\right) E^{-}\left(-\gamma_{2}, z_{2}\right)\left(-\left[\beta_{1}\left(z_{1}\right), \beta_{2}\left(z_{2}\right)\right] g_{-\left(\gamma_{1}, \gamma_{2}\right)}\left(z_{1}, z_{2}\right)\right. \\
\left.+\beta_{1}\left(z_{1}\right) \beta_{2}\left(z_{2}\right) g_{-\left(\gamma_{1}, \gamma_{2}\right)}\left(z_{1}, z_{2}\right)\right) E^{+}\left(-\gamma_{1}, z_{1}\right) E^{+}\left(-\gamma_{2}, z_{2}\right) e^{\gamma_{1}} e^{\gamma_{2}} z_{1}^{\gamma_{1}} z_{2}^{\gamma_{2}} .
\end{gathered}
$$

Similarly, we have

$$
\begin{gathered}
X^{\left(\beta_{1}\right)}\left(\gamma_{1}, z_{1}\right) X^{\left(\beta_{2}\right)}\left(\gamma_{2}, z_{2}\right) \\
=z_{1}^{\left(\gamma_{1}, \gamma_{1}\right) / 2} z_{2}^{\left(\gamma_{2}, \gamma_{2}\right) / 2} E^{-}\left(-\gamma_{1}, z_{1}\right) E^{-}\left(-\gamma_{2}, z_{2}\right) \beta_{1}\left(z_{1}\right) \beta_{2}\left(z_{2}\right) E^{+}\left(-\gamma_{1}, z_{1}\right) E^{+}\left(-\gamma_{2}, z_{2}\right) \\
\cdot e^{\gamma_{1}} e^{\gamma_{2}} z_{1}^{\gamma_{1}} z_{2}^{\gamma_{2}} z_{1}^{\left(\gamma_{1}, \gamma_{2}\right)}\left(1-\frac{z_{2}}{z_{1}}\right)^{\left(\gamma_{1}, \gamma_{2}\right)} \\
=z_{1}^{\left(\gamma_{1}, \gamma_{1}\right) / 2} z_{2}^{\left(\gamma_{2}, \gamma_{2}\right) / 2} E^{-}\left(-\gamma_{1}, z_{1}\right) E^{-}\left(-\gamma_{2}, z_{2}\right)\left(\beta_{1}\left(z_{1}\right) \beta_{2}\left(z_{2}\right) f_{-\left(\gamma_{1}, \gamma_{2}\right)}\left(z_{1}, z_{2}\right)\right) \\
\cdot E^{+}\left(-\gamma_{1}, z_{1}\right) E^{+}\left(-\gamma_{2}, z_{2}\right) e^{\gamma_{1}} e^{\gamma_{2}} z_{1}^{\gamma_{1}} z_{2}^{\gamma_{2}} .
\end{gathered}
$$


Therefore, we obtain

$$
\begin{aligned}
& {\left[X^{\left(\beta_{2}\right)}\left(\gamma_{2}, z_{2}\right), X^{\left(\beta_{1}\right)}\left(\gamma_{1}, z_{1}\right)\right]=X^{\left(\beta_{2}\right)}\left(\gamma_{2}, z_{2}\right) X^{\left(\beta_{1}\right)}\left(\gamma_{1}, z_{1}\right)} \\
& \quad-X^{\left(\beta_{1}\right)}\left(\gamma_{1}, z_{1}\right) X^{\left(\beta_{2}\right)}\left(\gamma_{2}, z_{2}\right) \\
& =z_{1}^{\left(\gamma_{1}, \gamma_{1}\right) / 2} z_{2}^{\left(\gamma_{2}, \gamma_{2}\right) / 2} \\
& \quad \cdot E^{-}\left(-\gamma_{1}, z_{1}\right) E^{-}\left(-\gamma_{2}, z_{2}\right)\left\{-\left[\beta_{1}\left(z_{1}\right), \beta_{2}\left(z_{2}\right)\right] g_{-\left(\gamma_{1}, \gamma_{2}\right)}\left(z_{1}, z_{2}\right)\right. \\
& \left.\quad-\beta_{1}\left(z_{1}\right) \beta_{2}\left(z_{2}\right)\left(f-\left(\gamma_{1}, \gamma_{2}\right)\left(z_{1}, z_{2}\right)-g_{-\left(\gamma_{1}, \gamma_{2}\right)}\left(z_{1}, z_{2}\right)\right)\right\} \\
& \quad \cdot E^{+}\left(-\gamma_{1}, z_{1}\right) E^{+}\left(-\gamma_{2}, z_{2}\right) e^{\gamma_{1}} e^{\gamma_{2}} z_{1}^{\gamma_{1}} z_{2}^{\gamma_{2}},
\end{aligned}
$$

where

$$
\begin{gathered}
{\left[\beta_{1}\left(z_{1}\right), \beta_{2}\left(z_{2}\right)\right] g_{-\left(\gamma_{1}, \gamma_{2}\right)}\left(z_{1}, z_{2}\right)+\beta_{1}\left(z_{1}\right) \beta_{2}\left(z_{2}\right)\left(f_{-\left(\gamma_{1}, \gamma_{2}\right)}\left(z_{1}, z_{2}\right)-g_{-\left(\gamma_{1}, \gamma_{2}\right)}\left(z_{1}, z_{2}\right)\right.} \\
=\left[\beta_{1}\left(z_{1}\right), \beta_{2}\left(z_{2}\right)\right] F_{1} G_{2}+F_{1} F_{2} \beta_{1}\left(z_{1}\right) \beta_{2}\left(z_{2}\right) .
\end{gathered}
$$

This proves the result for the case $k=2$. Now we prove this proposition by induction on $k \geq 2$. First, we compute by applying (3.35) and (3.36)

$$
\begin{gathered}
X^{\left(\beta_{k+1}\right)}\left(\gamma_{k+1}, z_{k+1}\right)\left[X^{\left(\beta_{k}\right)}\left(\gamma_{k}, z_{k}\right), \cdots, X^{\left(\beta_{1}\right)}\left(\gamma_{1}, z_{1}\right)\right] \\
=z_{k+1}^{\left(\gamma_{k+1}, \gamma_{k+1}\right) / 2} E^{-}\left(-\gamma_{k+1}, z_{k+1}\right) \beta_{k+1}\left(z_{k+1}\right) E^{+}\left(-\gamma_{k+1}, z_{k+1}\right) e^{\gamma_{k+1}} z_{k+1}^{\gamma_{k+1}} \\
\cdot(-1)^{k-1}\left(\prod_{i=1}^{k} z_{i}^{\left(\gamma_{i}, \gamma_{i}\right) / 2}\right)\left(\prod_{i=1}^{k} E^{-}\left(-\gamma_{i}, z_{i}\right)\right) Q\left(z_{1}, \cdots, z_{k}, \beta_{1}, \cdots, \beta_{k}\right)\left(\prod_{i=1}^{k} E^{+}\left(-\gamma_{i}, z_{i}\right)\right) \\
\cdot\left(e^{\gamma_{1}} \cdots e^{\gamma_{k}}\right)\left(\prod_{i=1}^{k} z_{i}^{\gamma_{i}}\right) \\
=(-1)^{k-1}\left(\prod_{i=1}^{k+1} z_{i}^{\left(\gamma_{i}, \gamma_{i}\right) / 2}\right)\left(\prod_{i=1}^{k+1} E^{-}\left(-\gamma_{i}, z_{i}\right)\right) \beta_{k+1}\left(z_{k+1}\right) Q\left(z_{1}, \cdots, z_{k}, \beta_{1}, \cdots, \beta_{k}\right) \\
\cdot\left(\prod_{i=1}^{k+1} E^{+}\left(-\gamma_{i}, z_{i}\right)\right)\left(e^{\gamma_{1}} \cdots e^{\gamma_{k+1}}\right)\left(\prod_{i=1}^{k+1} z_{i}^{\gamma_{i}}\right) \prod_{i=1}^{k}\left\{\left(1-z_{i} / z_{k+1}\right)^{\left(\gamma_{i}, \gamma_{k+1}\right)}\left(-z_{k+1}\right)^{\left(\gamma_{i}, \gamma_{k+1}\right)}\right\} .
\end{gathered}
$$

Similarly, we have

$$
\begin{gathered}
{\left[X^{\left(\beta_{k}\right)}\left(\gamma_{k}, z_{k}\right), \cdots, X^{\left(\beta_{1}\right)}\left(\gamma_{1}, z_{1}\right)\right] X^{\left(\beta_{k+1}\right)}\left(\gamma_{k+1}, z_{k+1}\right)} \\
=(-1)^{k-1}\left(\prod_{i=1}^{k+1} z_{i}^{\left(\gamma_{i}, \gamma_{i}\right) / 2}\right)\left(\prod_{i=1}^{k+1} E^{-}\left(-\gamma_{i}, z_{i}\right)\right) Q\left(z_{1}, \cdots, z_{k}, \beta_{1}, \cdots, \beta_{k}\right) \beta_{k+1}\left(z_{k+1}\right) \\
\cdot\left(\prod_{i=1}^{k+1} E^{+}\left(-\gamma_{i}, z_{i}\right)\right)\left(e^{\gamma_{1}} \cdots e^{\gamma_{k+1}}\right)\left(\prod_{i=1}^{k+1} z_{i}^{\gamma_{i}}\right) \prod_{i=1}^{k}\left\{\left(1-z_{k+1} / z_{i}\right)^{\left(\gamma_{i}, \gamma_{k+1}\right)} z_{i}^{\left(\gamma_{i}, \gamma_{k+1}\right)}\right\} .
\end{gathered}
$$

Therefore, we obtain

$$
\begin{gathered}
{\left[X^{\left(\beta_{k+1}\right)}\left(\gamma_{k+1}, z_{k+1}\right), \cdots, X^{\left(\beta_{1}\right)}\left(\gamma_{1}, z_{1}\right)\right]} \\
=(-1)^{k-1}\left(\prod_{i=1}^{k+1} z_{i}^{\left(\gamma_{i}, \gamma_{i}\right) / 2}\right)\left(\prod_{i=1}^{k+1} E^{-}\left(-\gamma_{i}, z_{i}\right)\right) \tilde{Q}\left(\prod_{i=1}^{k+1} E^{+}\left(-\gamma_{i}, z_{i}\right)\right)\left(e^{\gamma_{1}} \cdots e^{\gamma_{k+1}}\right)\left(\prod_{i=1}^{k+1} z_{i}^{\gamma_{i}}\right),
\end{gathered}
$$

where 


$$
\begin{gathered}
\tilde{Q}=\left(\prod_{i=1}^{k} g_{-\left(\gamma_{i}, \gamma_{k+1}\right)}\left(z_{i}, z_{k+1}\right)\right) \beta_{k+1}\left(z_{k+1}\right) Q\left(z_{1}, \cdots, z_{k}, \beta_{1}, \cdots, \beta_{k}\right) \\
-\left(\prod_{i=1}^{k} f_{-\left(\gamma_{i}, \gamma_{k+1}\right)}\left(z_{i}, z_{k+1}\right)\right) Q\left(z_{1}, \cdots, z_{k}, \beta_{1}, \cdots, \beta_{k}\right) \beta_{k+1}\left(z_{k+1}\right) \\
=(-1)\left\{\left(\prod_{i=1}^{k} f_{-\left(\gamma_{i}, \gamma_{k+1}\right)}\left(z_{i}, z_{k+1}\right)\right) Q\left(z_{1}, \cdots, z_{k}, \beta_{1}, \cdots, \beta_{k}\right) \beta_{k+1}\left(z_{k+1}\right)\right. \\
\left.-\left(\prod_{i=1}^{k} g_{-\left(\gamma_{i}, \gamma_{k+1}\right)}\left(z_{i}, z_{k+1}\right)\right) \beta_{k+1}\left(z_{k+1}\right) Q\left(z_{1}, \cdots, z_{k}, \beta_{1}, \cdots, \beta_{k}\right)\right\}
\end{gathered}
$$

equals a linear combination of terms of the form

$$
\begin{gathered}
(-1)\left\{\left(\prod_{i=1}^{k} f_{-\left(\gamma_{i}, \gamma_{k+1}\right)}\left(z_{i}, z_{k+1}\right)\right) \prod_{m=1}^{l}\left(\left[\beta_{i_{m}}\left(z_{i_{m}}\right), \beta_{j_{m}}\left(z_{j_{m}}\right)\right] F_{i_{m}} G_{j_{m}}\right)\left(\prod_{n=1}^{s} F_{t_{n}}\right)\right. \\
\cdot\left(\beta_{t_{1}}\left(z_{t_{1}}\right) \cdots \beta_{t_{s}}\left(z_{t_{s}}\right)\right) \beta_{k+1}\left(z_{k+1}\right) \\
-\left(\prod_{i=1}^{k} g_{-\left(\gamma_{i}, \gamma_{k+1}\right)}\left(z_{i}, z_{k+1}\right)\right) \beta_{k+1}\left(z_{k+1}\right) \prod_{m=1}^{l}\left(\left[\beta_{i_{m}}\left(z_{i_{m}}\right), \beta_{j_{m}}\left(z_{j_{m}}\right)\right] F_{i_{m}} G_{j_{m}}\right)\left(\prod_{n=1}^{s} F_{t_{n}}\right) \\
\left.\cdot\left(\beta_{t_{1}}\left(z_{t_{1}}\right) \cdots \beta_{t_{s}}\left(z_{t_{s}}\right)\right)\right\}
\end{gathered}
$$

where

$$
\begin{gathered}
\beta_{k+1}\left(z_{k+1}\right)\left(\beta_{t_{1}}\left(z_{t_{1}}\right) \cdots \beta_{t_{s}}\left(z_{t_{s}}\right)\right)=\beta_{t_{1}}\left(z_{t_{1}}\right) \cdots \beta_{t_{s}}\left(z_{t_{s}}\right) \beta_{k+1}\left(z_{k+1}\right) \\
\left.-\sum_{p=1}^{s}\left[\beta_{t_{p}}\left(z_{t_{p}}\right), \beta_{k+1}\left(z_{k+1}\right)\right]\left(\beta_{t_{1}}\left(z_{t_{1}}\right) \cdots \widehat{\beta_{t_{p}}\left(z_{t_{p}}\right.}\right) \cdots \beta_{t_{s}}\left(z_{t_{s}}\right)\right),
\end{gathered}
$$

where ' $\hat{\prime}$ ' means the term is deleted.

Therefore, we have $\tilde{Q}=$ a linear combination of terms of the form

$$
\begin{aligned}
&(-1)\left\{\prod_{m=1}^{l}\left(\left[\beta_{i_{m}}\left(z_{i_{m}}\right), \beta_{j_{m}}\left(z_{j_{m}}\right)\right] F_{i_{m}} G_{j_{m}}\right)\right. \\
& \cdot\left(\prod_{n=1}^{s} F_{t_{n}}\right) F_{k+1}\left(\beta_{t_{1}}\left(z_{t_{1}}\right) \cdots \beta_{t_{s}}\left(z_{t_{s}}\right)\right) \beta_{k+1}\left(z_{k+1}\right) \\
&+\sum_{p=1}^{s}\left(\prod_{m=1}^{l}\left(\left[\beta_{i_{m}}\left(z_{i_{m}}\right), \beta_{j_{m}}\left(z_{j_{m}}\right)\right] F_{i_{m}} G_{j_{m}}\right)\right)\left(\left[\beta_{t_{p}}\left(z_{t_{p}}\right), \beta_{k+1}\left(z_{k+1}\right)\right] F_{t_{p}} G_{k+1}\right) \\
&\left.\left.\cdot\left(\prod_{\substack{n=1 \\
n \neq p}}^{s} F_{t_{n}}\right)\left(\beta_{t_{1}}\left(z_{t_{1}}\right) \cdots \widehat{\beta_{t_{p}}\left(z_{t_{p}}\right.}\right) \cdots \beta_{t_{s}}\left(z_{t_{s}}\right)\right)\right\}
\end{aligned}
$$

as required.

Lemma 3.38. Suppose $\beta_{i_{m}}=\alpha_{i}^{+}, \beta_{j_{m}}=\alpha_{i}^{-}$, and $\gamma_{i_{m}}=\alpha_{i}, \gamma_{j_{m}}=-\alpha_{i}$, for some $i \in J$. Then 


$$
\begin{aligned}
& {\left[\beta_{i_{m}}\left(z_{i_{m}}\right), \beta_{j_{m}}\left(z_{j_{m}}\right)\right] g_{-\left(\gamma_{i_{m}}, \gamma_{j_{m}}\right)}\left(z_{i_{m}}, z_{j_{m}}\right)} \\
& \quad=\left(z_{i_{m}}^{-\left(\gamma_{i_{m}}, \gamma_{i_{m}}\right) / 2} z_{j_{m}}^{-\left(\gamma_{j_{m}}, \gamma_{j_{m}}\right) / 2}\right)(D \delta)\left(z_{j_{m}} / z_{i_{m}}\right) .
\end{aligned}
$$

Proof. Let $n=\left(\gamma_{i_{m}}, \gamma_{j_{m}}\right) / 2=-\left(\alpha_{i}, \alpha_{i}\right) / 2$, then $n \in \mathbf{N}$, and

$$
g_{-\left(\gamma_{i_{m}}, \gamma_{j_{m}}\right)}\left(z_{i_{m}}, z_{j_{m}}\right)=\left(-z_{j_{m}}\right)^{\left(\gamma_{i_{m}}, \gamma_{j_{m}}\right)}\left(1-z_{i_{m}} / z_{j_{m}}\right)^{\left(\gamma_{i_{m}}, \gamma_{j_{m}}\right)}=\left(z_{i_{m}}-z_{j_{m}}\right)^{2 n}
$$

and

$$
\left[\beta_{i_{m}}\left(z_{i_{m}}\right), \beta_{j_{m}}\left(z_{j_{m}}\right)\right]=\left[\alpha_{i}^{+}\left(z_{i_{m}}\right), \alpha_{i}^{-}\left(z_{j_{m}}\right)\right]=\sum_{k, l \in \mathbf{Z}}\left[\alpha_{i}^{+}(k), \alpha_{i}^{-}(l)\right] z_{i_{m}}^{-k} z_{j_{m}}^{-l} .
$$

Thus, we have

$$
\begin{gathered}
{\left[\beta_{i_{m}}\left(z_{i_{m}}\right), \beta_{j_{m}}\left(z_{j_{m}}\right)\right] g_{-\left(\gamma_{i_{m}}, \gamma_{j_{m}}\right)}\left(z_{i_{m}}, z_{j_{m}}\right)} \\
=\left(z_{i_{m}}-z_{j_{m}}\right)^{2 n} \sum_{k, l \in \mathbf{Z}}\left[\alpha_{i}^{+}(k), \alpha_{i}^{-}(l)\right] z_{i_{m}}^{-k} z_{j_{m}}^{-l} \\
=\sum_{j=0}^{2 n}\left(\begin{array}{c}
2 n \\
j
\end{array}\right) z_{i_{m}}^{j}\left(-z_{j_{m}}\right)^{2 n-j} \sum_{k, l \in \mathbf{Z}}\left[\alpha_{i}^{+}(k), \alpha_{i}^{-}(l)\right] z_{i_{m}}^{-k} z_{j_{m}}^{-l} \\
=\sum_{j=0}^{2 n} \sum_{k, l \in \mathbf{Z}}(-1)^{j}\left(\begin{array}{c}
2 n \\
j
\end{array}\right)\left[\alpha_{i}^{+}(k), \alpha_{i}^{-}(l)\right] z_{i_{m}}^{j-k} z_{j_{m}}^{2 n-j-l} \\
=\sum_{j=0}^{2 n} \sum_{k, l \in \mathbf{Z}}(-1)^{j}\left(\begin{array}{c}
2 n \\
j
\end{array}\right)\left(\alpha_{i}^{+}, \alpha_{i}^{-}\right) \zeta_{\alpha_{i}}(k) \delta_{k+l, 0} z_{i_{m}}^{j-k} z_{j_{m}}^{2 n-j-l} \\
=\sum_{j=0}^{2 n} \sum_{k \in \mathbf{Z}}(-1)^{j}\left(\begin{array}{c}
2 n \\
j
\end{array}\right) \zeta_{\alpha_{i}}(k) z_{i_{m}}^{j-k} z_{j_{m}}^{2 n-j+k} \\
=z_{i_{m}}^{n} z_{j_{m}}^{n} \sum_{j=0}^{2 n} \sum_{k \in \mathbf{Z}}(-1)^{j}\left(\begin{array}{c}
2 n \\
j
\end{array}\right) \zeta_{\alpha_{i}}(k) z_{i_{m}}^{j-k-n} z_{j_{m}}^{n-j+k} \\
=z_{i_{m}}^{n} z_{j_{m}}^{n} \sum_{j=0}^{2 n} \sum_{k \in \mathbf{Z}}(-1)^{j}\left(\begin{array}{c}
2 n \\
j
\end{array}\right) \zeta_{\alpha_{i}}(k-n+j)\left(z_{j_{m}} / z_{i_{m}}\right)^{k} .
\end{gathered}
$$

By Lemma 3.4, this gives us

$$
\begin{gathered}
{\left[\beta_{i_{m}}\left(z_{i_{m}}\right), \beta_{j_{m}}\left(z_{j_{m}}\right)\right] g_{-\left(\gamma_{i_{m}}, \gamma_{j_{m}}\right)}\left(z_{i_{m}}, z_{j_{m}}\right)} \\
=z_{i_{m}}^{n} z_{j_{m}}^{n} \sum_{k \in \mathbf{Z}} k\left(z_{j_{m}} / z_{i_{m}}\right)^{k}=z_{i_{m}}^{n} z_{j_{m}}^{n}(D \delta)\left(z_{j_{m}} / z_{i_{m}}\right),
\end{gathered}
$$

as required.

Corollary 3.39. Let $i \in J$. We have

$$
\begin{aligned}
& {\left[X^{\left(\alpha_{i}^{+}\right)}\left(\alpha_{i}, z\right), X^{\left(\alpha_{i}^{-}\right)}\left(-\alpha_{i}, w\right)\right]} \\
& \quad=(-1)^{\frac{1}{2}\left(\alpha_{i}, \alpha_{i}\right)}\left((D \delta)\left(\frac{w}{z}\right) \not \varnothing+\delta\left(\frac{w}{z}\right) \alpha_{i}(w)\right) .
\end{aligned}
$$


Proof. Set $\left(\alpha_{i}, \alpha_{i}\right)=-2 n$, then $n \in \mathbf{N}$, and note

$$
f_{\left(\alpha_{i}, \alpha_{i}\right)}(w, z)-g_{\left(\alpha_{i}, \alpha_{i}\right)}(w, z)=0, \quad g_{\left(\alpha_{i}, \alpha_{i}\right)}(w, z)=g_{\left(\alpha_{i}, \alpha_{i}\right)}(z, w) .
$$

By Lemma 3.38, we have

$$
\left[\alpha_{i}^{+}(z), \alpha_{i}^{-}(w)\right] g_{\left(\alpha_{i}, \alpha_{i}\right)}(z, w)=z^{n} w^{n}(D \delta)\left(\frac{w}{z}\right) .
$$

Thus by (3.37), (3.41) and (3.42) we obtain

$$
\begin{gathered}
{\left[X^{\left(\alpha_{i}^{+}\right)}\left(\alpha_{i}, z\right), X^{\left(\alpha_{i}^{-}\right)}\left(-\alpha_{i}, w\right)\right]} \\
=z^{-n} w^{-n} E^{-}\left(\alpha_{i}, w\right) E^{-}\left(-\alpha_{i}, z\right)\left(\left[\alpha_{i}^{+}(z), \alpha_{i}^{-}(w)\right] g_{\left(\alpha_{i}, \alpha_{i}\right)}(w, z)\right) \\
\cdot E^{+}\left(\alpha_{i}, w\right) E^{+}\left(-\alpha_{i}, z\right) e^{-\alpha_{i}} e^{\alpha_{i}} w^{-\alpha_{i}} z^{\alpha_{i}} \\
=E^{-}\left(\alpha_{i}, w\right) E^{-}\left(-\alpha_{i}, z\right) E^{+}\left(\alpha_{i}, w\right) E^{+}\left(-\alpha_{i}, z\right) e^{-\alpha_{i}} e^{\alpha_{i}} w^{-\alpha_{i}} z^{\alpha_{i}}(D \delta)\left(\frac{w}{z}\right) \\
=(-1)^{\left(\alpha_{i}, \alpha_{i}\right) / 2} E^{-}\left(\alpha_{i}, w\right) E^{-}\left(-\alpha_{i}, z\right) E^{+}\left(\alpha_{i}, w\right) E^{+}\left(-\alpha_{i}, z\right) w^{-\alpha_{i}} z^{\alpha_{i}}(D \delta)\left(\frac{w}{z}\right) \\
=(-1)^{\frac{1}{2}\left(\alpha_{i}, \alpha_{i}\right)}\left((D \delta)\left(\frac{w}{z}\right) \phi+\delta\left(\frac{w}{z}\right) \alpha_{i}(w)\right),
\end{gathered}
$$

where in the last step we have used Lemma 3.10.

Corollary 3.43. Let $Q\left(z_{1}, \cdots, z_{k}, \beta_{1}, \cdots, \beta_{k}\right)$ be as in Proposition 3.32. We suppose that $\beta_{1}, \ldots, \beta_{k} \in\left\{\alpha_{j}^{+}, \alpha_{j}^{-}, \not \mid j \in J\right\}, \gamma_{1}, \ldots, \gamma_{k} \in \Gamma$, and assume $\gamma_{i}=\alpha_{j}$ if $\beta_{i}=\alpha_{j}^{+}$for some $j \in J$, or $\gamma_{i}=-\alpha_{j}$ if $\beta_{i}=\alpha_{j}^{-}$for some $j \in J$, for $1 \leq i \leq k$. Then $Q\left(z_{1}, \cdots, z_{k}, \beta_{1}, \cdots, \beta_{k}\right)=$ a linear combination of terms of the form

$$
\begin{gathered}
\left(\prod_{m=1}^{l}\left(z_{i_{m}}^{-\left(\gamma_{i_{m}}, \gamma_{i_{m}}\right) / 2} z_{j_{m}}^{-\left(\gamma_{j_{m}}, \gamma_{j_{m}}\right) / 2}\right)\right)\left\{\prod _ { m = 1 } ^ { l } \left((D \delta)\left(z_{j_{m}} / z_{i_{m}}\right)\right.\right. \\
\left.\left.\cdot\left(\prod_{\substack{\tau=1 \\
\tau \neq i_{m}}}^{j_{m}-1} g_{-\left(\gamma_{\tau}, \gamma_{j_{m}}\right)}\left(z_{\tau}, z_{j_{m}}\right)\right) F_{i_{m}}\right)\right\}\left(\prod_{n=1}^{s} F_{t_{n}}\right)\left(\beta_{t_{1}}\left(z_{1}\right) \cdots \beta_{t_{s}}\left(z_{s}\right)\right),
\end{gathered}
$$

where $\left(i_{1}, \ldots, i_{l}, j_{1}, \ldots, j_{l}, t_{1}, \ldots t_{s}\right)$ is some permutation of $\{1,2, \ldots, k\}$ given in Proposition 3.32.

Proof. This follows immediately from Lemma 3.38.

Corollary 3.45. Let $\gamma_{1}, \ldots, \gamma_{k} \in \Gamma, \beta_{1}, \ldots, \beta_{k} \in L \oplus \mathbf{Z} \not, \quad k \geq 2$. Suppose $\left(\beta_{i}, \beta_{j}\right)=0$ for all $i \neq j$, and $-\sum_{1 \leq i<j \leq k}\left(\gamma_{i}, \gamma_{j}\right)<k-1$. Then

$$
\left[X^{\left(\beta_{k}\right)}\left(\gamma_{k}, z_{k}\right), \cdots, X^{\left(\beta_{1}\right)}\left(\gamma_{1}, z_{1}\right)\right]=0,
$$

or equivalently $\left[x_{n_{k}}^{\left(\beta_{k}\right)}\left(\gamma_{k}\right), \ldots, x_{n_{1}}^{\left(\beta_{1}\right)}\left(\gamma_{1}\right)\right]=0$, for all $n_{1}, \ldots, n_{k} \in \mathbf{Z}$.

Proof. This follows from (3.34), Proposition 3.32 and Theorem 3.22.

Notice that if $\gamma_{1}, \ldots, \gamma_{k} \in \Gamma$ satisfy $\left(\gamma_{i}, \gamma_{i}\right) \leq 2$ for $i=1,2, \ldots, k,(k \geq 2)$, then $\left(\sum_{i=1}^{k} \gamma_{i}, \sum_{i=1}^{k} \gamma_{i}\right)>2$ implies $-\sum_{1 \leq i<j \leq k}\left(\gamma_{i}, \gamma_{j}\right)<k-1$. The converse statement is true only if $\left(\gamma_{i}, \gamma_{i}\right)=2$ for all $i$. We have 
Corollary 3.46. Let $\gamma_{1}, \ldots, \gamma_{k} \in \Gamma, \beta_{1}, \ldots, \beta_{k} \in L \oplus \mathbf{Z} \not \phi, \quad k \geq 2$. Suppose $\left(\gamma_{i}, \gamma_{i}\right) \leq 2$ for $i=1,2, \ldots, k,\left(\beta_{i}, \beta_{j}\right)=0$ for all $i \neq j$, and $\left(\sum_{i=1}^{k} \gamma_{i}, \sum_{i=1}^{k} \gamma_{i}\right)>2$. Then

$$
\left[X^{\left(\beta_{k}\right)}\left(\gamma_{k}, z_{k}\right), \cdots, X^{\left(\beta_{1}\right)}\left(\gamma_{1}, z_{1}\right)\right]=0,
$$

or equivalently $\left[x_{n_{k}}^{\left(\beta_{k}\right)}\left(\gamma_{k}\right), \ldots, x_{n_{1}}^{\left(\beta_{1}\right)}\left(\gamma_{1}\right)\right]=0$, for all $n_{1}, \ldots, n_{k} \in \mathbf{Z}$.

We need one more result to deal with the case when not all of $\beta_{1}, \ldots, \beta_{k}$ are pairwise orthogonal.

Theorem 3.47. Let $k \geq 2$, and let $\left(i_{1}, \ldots, i_{l}, j_{1}, \ldots, j_{l}, t_{1}, \ldots, t_{s}\right)$ be any permutation of $\{1,2, \ldots, k\}$ given in Proposition 3.32. Let $\gamma_{1}, \ldots, \gamma_{k} \in \Gamma$ be such that $\left(\gamma_{\tau}, \gamma_{\tau}\right) \leq 2$ and $\gamma_{i_{m}}+\gamma_{j_{m}}=0$ for $1 \leq \tau \leq k, 1 \leq m \leq l$. Then

$$
\bar{Q}\left(z_{1}, \cdots, z_{k}\right):=\prod_{m=1}^{l}\left((D \delta)\left(z_{j_{m}} / z_{i_{m}}\right)\left\{\prod_{\substack{\tau=1 \\ \tau \neq i_{m}}}^{j_{m}-1} g_{-\left(\gamma_{\tau}, \gamma_{j_{m}}\right)}\left(z_{\tau}, z_{j_{m}}\right) F_{i_{m}}\right\}\right) \prod_{n=1}^{s} F_{t_{n}}=0,
$$

provided $\left(\sum_{i=1}^{k} \gamma_{i}, \sum_{i=1}^{k} \gamma_{i}\right)>2$

Proof. Set $\lambda_{i j}=-\left(\gamma_{i}, \gamma_{j}\right), i, j=1,2, \ldots, k$. Then $\left(\sum_{i=1}^{k} \gamma_{i}, \sum_{i=1}^{k} \gamma_{i}\right)>2$ implies $\sum_{1 \leq i<j \leq k} \lambda_{i j}<k-1$. If $k=2$, then $l$ must be zero and $s=2$, thus

$$
\bar{Q}\left(z_{1}, z_{2}\right)=\prod_{n=1}^{2} F_{n}=f_{-\left(\gamma_{1}, \gamma_{2}\right)}\left(z_{1}, z_{2}\right)-g_{-\left(\gamma_{1}, \gamma_{2}\right)}\left(z_{1}, z_{2}\right)
$$

which is zero by Theorem 3.22 whenever $\left(\gamma_{1}+\gamma_{2}, \gamma_{1}+\gamma_{2}\right)>2$. Now we prove (3.48) by induction on $k$ and so we assume $k \geq 3$. We divide the argument into two cases.

Case 1. $l=0$.

In this case, we have $\bar{Q}\left(z_{1}, \cdots, z_{k}\right)=\prod_{n=1}^{k} F_{n}$, which is zero by Theorem 3.22 .

Case 2. $\quad l \geq 1$.

For this case, we divide the proof into two subcases.

Subcase A. $l=1$ and $i_{1}=1$.

In this case, $\bar{Q}\left(z_{1}, \ldots, z_{k}\right)$ can be written as follows:

$$
\bar{Q}\left(z_{1}, \ldots, z_{k}\right)=(D \delta)\left(z_{j_{1}} / z_{1}\right)\left(\prod_{\tau=2}^{j_{1}-1} g_{-\left(\gamma_{\tau}, \gamma_{j_{1}}\right)}\left(z_{\tau}, z_{j_{1}}\right)\right) \prod_{\substack{n=2 \\ n \neq j_{1}}}^{k} F_{n}
$$

If $j_{1}=2$, then $\gamma_{1}+\gamma_{2}=\gamma_{i_{1}}+\gamma_{j_{1}}=0$. Thus we have

$$
\bar{Q}\left(z_{1}, \ldots, z_{k}\right)=(D \delta)\left(\frac{z_{2}}{z_{1}}\right) \prod_{n=3}^{k} F_{n} .
$$


We note that

$$
\begin{gathered}
(D \delta)\left(\frac{z_{2}}{z_{1}}\right) F_{3} \\
=(D \delta)\left(\frac{z_{2}}{z_{1}}\right)\left(f_{-\left(\gamma_{1}, \gamma_{3}\right)}\left(z_{1}, z_{3}\right) f_{-\left(\gamma_{2}, \gamma_{3}\right)}\left(z_{2}, z_{3}\right)-g_{-\left(\gamma_{1}, \gamma_{3}\right)}\left(z_{1}, z_{3}\right) g_{-\left(\gamma_{2}, \gamma_{3}\right)}\left(z_{2}, z_{3}\right)\right) \\
=(D \delta)\left(\frac{z_{2}}{z_{1}}\right)\left(f_{-\left(\gamma_{1}, \gamma_{3}\right)}\left(z_{2}, z_{3}\right) f_{-\left(\gamma_{2}, \gamma_{3}\right)}\left(z_{2}, z_{3}\right)-g_{-\left(\gamma_{1}, \gamma_{3}\right)}\left(z_{2}, z_{3}\right) g_{-\left(\gamma_{2}, \gamma_{3}\right)}\left(z_{2}, z_{3}\right)\right) \\
+\delta\left(\frac{z_{2}}{z_{1}}\right)\left(D_{z_{1}} f_{-\left(\gamma_{1}, \gamma_{3}\right)}\left(z_{1}, z_{3}\right) f_{-\left(\gamma_{2}, \gamma_{3}\right)}\left(z_{2}, z_{3}\right)-D_{z_{1}} g_{-\left(\gamma_{1}, \gamma_{3}\right)}\left(z_{1}, z_{3}\right) g_{-\left(\gamma_{2}, \gamma_{3}\right)}\left(z_{2}, z_{3}\right)\right) \\
=\left(\gamma_{1}, \gamma_{3}\right) z_{2} \delta\left(\frac{z_{2}}{z_{1}}\right)\left(f_{1}\left(z_{2}, z_{3}\right)-g_{1}\left(z_{2}, z_{3}\right)\right)=\left(\gamma_{1}, \gamma_{3}\right) \delta\left(\frac{z_{2}}{z_{1}}\right) \delta\left(z_{2} / z_{3}\right) .
\end{gathered}
$$

Therefore, by using the identity $\left.\left(f_{-\left(\gamma_{1}, \gamma_{j}\right)}\left(z_{1}, z_{j}\right) f_{-\left(\gamma_{2}, \gamma_{j}\right)}\left(z_{2}, z_{j}\right)\right)\right|_{z_{1}=z_{2}}=1$ and the same identity for the $g$ 's, we obtain from (3.50)

$$
\begin{gathered}
\bar{Q}\left(z_{1}, \ldots, z_{k}\right)=\left(\gamma_{1}, \gamma_{3}\right) \delta\left(\frac{z_{2}}{z_{1}}\right) \delta\left(z_{2} / z_{3}\right) \prod_{n=4}^{k} F_{n} \\
=\left.\left(\gamma_{1}, \gamma_{3}\right) \delta\left(\frac{z_{2}}{z_{1}}\right) \delta\left(z_{2} / z_{3}\right)\left(\prod_{n=4}^{k} F_{n}\right)\right|_{z_{1}=z_{2}}=\left(\gamma_{1}, \gamma_{3}\right) \delta\left(\frac{z_{2}}{z_{1}}\right) \delta\left(z_{2} / z_{3}\right) \bar{Q}\left(z_{3}, z_{4}, \ldots, z_{k}\right),
\end{gathered}
$$

which is zero by induction. Thus, we may assume $j_{1} \geq 3$ in (3.49). We note that $\left(\gamma_{2}, \gamma_{j_{1}}\right)+\left(\gamma_{1}, \gamma_{2}\right)=0$ and

$$
\begin{gathered}
(D \delta)\left(z_{j_{1}} / z_{1}\right) g_{-\left(\gamma_{2}, \gamma_{j_{1}}\right)}\left(z_{2}, z_{j_{1}}\right) F_{2} \\
=(D \delta)\left(z_{j_{1}} / z_{1}\right) g_{-\left(\gamma_{2}, \gamma_{j_{1}}\right)}\left(z_{2}, z_{j_{1}}\right)\left(f_{-\left(\gamma_{1}, \gamma_{2}\right)}\left(z_{1}, z_{2}\right)-g_{-\left(\gamma_{1}, \gamma_{2}\right)}\left(z_{1}, z_{2}\right)\right) \\
=-\left.\delta\left(z_{j_{1}} / z_{1}\right)\left(D_{z_{j_{1}}} g_{-\left(\gamma_{2}, \gamma_{j_{1}}\right)}\left(z_{2}, z_{j_{1}}\right)\right)\right|_{z_{j_{1}}=z_{1}}\left(f_{-\left(\gamma_{1}, \gamma_{2}\right)}\left(z_{1}, z_{2}\right)-g_{-\left(\gamma_{1}, \gamma_{2}\right)}\left(z_{1}, z_{2}\right)\right),
\end{gathered}
$$

where we have used Lemma 3.10, (3.17) and (3.18). This and Corollary 3.27 give us

$$
(D \delta)\left(z_{j_{1}} / z_{1}\right) g_{-\left(\gamma_{2}, \gamma_{j_{1}}\right)}\left(z_{2}, z_{j_{1}}\right) F_{2}=\frac{\left(\gamma_{2}, \gamma_{j_{1}}\right)+\left|\left(\gamma_{2}, \gamma_{j_{1}}\right)\right|}{2} \delta\left(z_{j_{1}} / z_{1}\right) \delta\left(\frac{z_{2}}{z_{1}}\right) .
$$

Therefore, we obtain

$$
\begin{gathered}
\bar{Q}\left(z_{1}, \ldots, z_{k}\right)=(D \delta)\left(z_{j_{1}} / z_{1}\right)\left(\prod_{\tau=2}^{j_{1}-1} g_{-\left(\gamma_{\tau}, \gamma_{j_{1}}\right)}\left(z_{\tau}, z_{j_{1}}\right)\right) \prod_{\substack{n=2 \\
n \neq j_{1}}}^{k} F_{n} \\
=\left.\left\{\left(\prod_{\tau=3}^{j_{1}-1} g_{-\left(\gamma_{\tau}, \gamma_{j_{1}}\right)}\left(z_{\tau}, z_{j_{1}}\right)\right) \prod_{\substack{n=3 \\
n \neq j_{1}}}^{k} F_{n}\right\}\right|_{z_{j_{1}}=z_{1}} \frac{\left(\gamma_{2}, \gamma_{j_{1}}\right)+\left|\left(\gamma_{2}, \gamma_{j_{1}}\right)\right|}{2} \delta\left(z_{j_{1}} / z_{1}\right) \delta\left(\frac{z_{2}}{z_{1}}\right) \\
=(-1)^{\left(\gamma_{1}, \sum_{3 \leq \tau<j_{1}} \gamma_{\tau}\right)} \frac{\left(\gamma_{2}, \gamma_{j_{1}}\right)+\left|\left(\gamma_{2}, \gamma_{j_{1}}\right)\right|}{2} \delta\left(z_{j_{1}} / z_{1}\right) \delta\left(\frac{z_{2}}{z_{1}}\right) \bar{Q}\left(z_{2}, \ldots, \hat{z}_{j_{1}}, \ldots, z_{k}\right),
\end{gathered}
$$

by (3.17) and (3.18) again. By induction this is zero as required.

Subcase B. $i_{p} \geq 2$ for some $1 \leq p \leq l$. 
We fix this $p$ and note that

$$
\begin{gathered}
(*):=(D \delta)\left(z_{j_{p}} / z_{i_{p}}\right)\left(\prod_{\substack{\tau=1 \\
\tau \neq i_{p}}}^{j_{p}-1} g_{-\left(\gamma_{\tau}, \gamma_{j_{p}}\right)}\left(z_{\tau}, z_{j_{p}}\right)\right) F_{i_{p}} \\
=(D \delta)\left(z_{j_{p}} / z_{i_{p}}\right)\left(\prod_{\substack{\tau=1 \\
\tau \neq i_{p}}}^{j_{p}-1} g_{\lambda_{\tau j_{p}}}\left(z_{\tau}, z_{j_{p}}\right)\right)\left(\prod_{\tau=1}^{i_{p}-1} f_{\lambda_{\tau i_{p}}}\left(z_{\tau}, z_{i_{p}}\right)-\prod_{\tau=1}^{i_{p}-1} g_{\lambda_{\tau i} p}\left(z_{\tau}, z_{i_{p}}\right)\right) \\
=(D \delta)\left(z_{j_{p}} / z_{i_{p}}\right)\left(\prod_{\tau=i_{p}+1}^{j_{p}-1} g_{\lambda_{\tau j_{p}}}\left(z_{\tau}, z_{j_{p}}\right)\right)\left(\prod_{\tau=1}^{i_{p}-1} g_{\lambda_{\tau j_{p}}}\left(z_{\tau}, z_{j_{p}}\right)\right) \\
\cdot\left(\prod_{\tau=1}^{i_{p}-1} f_{\lambda_{\tau i_{p}}}\left(z_{\tau}, z_{i_{p}}\right)-\prod_{\tau=1}^{i_{p}-1} g_{\lambda_{\tau i_{p}}}\left(z_{\tau}, z_{i_{p}}\right)\right) .
\end{gathered}
$$

By Lemma 3.10, this gives us

$$
\begin{aligned}
& (*)=(D \delta)\left(z_{j_{p}} / z_{i_{p}}\right)\left(\prod_{\tau=i_{p}+1}^{j_{p}-1} g_{\lambda_{\tau_{p}}}\left(z_{\tau}, z_{i_{p}}\right)\right)\left(\prod_{\tau=1}^{i_{p}-1} g_{\lambda_{\tau_{p}}}\left(z_{\tau}, z_{i_{p}}\right)\right) \\
& \cdot\left(\prod_{\tau=1}^{i_{p}-1} f_{\lambda_{\tau i_{p}}}\left(z_{\tau}, z_{i_{p}}\right)-\prod_{\tau=1}^{i_{p}-1} g_{\lambda_{\tau i_{p}}}\left(z_{\tau}, z_{i_{p}}\right)\right) \\
& -\left.\delta\left(z_{j_{p}} / z_{i_{p}}\right) D_{z_{j_{p}}}\left\{\left(\prod_{\tau=i_{p}+1}^{j_{p}-1} g_{\lambda_{\tau j_{p}}}\left(z_{\tau}, z_{j_{p}}\right)\right)\left(\prod_{\tau=1}^{i_{p}-1} g_{\lambda_{\tau j_{p}}}\left(z_{\tau}, z_{j_{p}}\right)\right)\right\}\right|_{z_{j_{p}}=z_{i_{p}}} \\
& \cdot\left(\prod_{\tau=1}^{i_{p}-1} f_{\lambda_{\tau i_{p}}}\left(z_{\tau}, z_{i_{p}}\right)-\prod_{\tau=1}^{i_{p}-1} g_{\lambda_{\tau i_{p}}}\left(z_{\tau}, z_{i_{p}}\right)\right)
\end{aligned}
$$

Note that $\lambda_{\tau i_{m}}+\lambda_{\tau j_{m}}=-\left(\gamma_{\tau}, \gamma_{i_{m}}+\gamma_{j_{m}}\right)=0$, thus by Lemma 3.28 we have

$$
\left(\prod_{\tau=1}^{i_{p}-1} g_{\lambda_{\tau j_{p}}}\left(z_{\tau}, z_{i_{p}}\right)\right)\left(\prod_{\tau=1}^{i_{p}-1} f_{\lambda_{\tau i_{p}}}\left(z_{\tau}, z_{i_{p}}\right)-\prod_{\tau=1}^{i_{p}-1} g_{\lambda_{\tau i_{p}}}\left(z_{\tau}, z_{i_{p}}\right)\right)=0
$$

and then by Lemma 3.30 we obtain

$$
\begin{gathered}
(*)=-\left.\delta\left(z_{j_{p}} / z_{i_{p}}\right)\left(\prod_{\tau=i_{p}+1}^{j_{p}-1} g_{\lambda_{\tau j_{p}}}\left(z_{\tau}, z_{i_{p}}\right)\right) D_{z_{j_{p}}}\left(\prod_{\tau=1}^{i_{p}-1} g_{\lambda_{\tau j_{p}}}\left(z_{\tau}, z_{j_{p}}\right)\right)\right|_{z_{j_{p}}=z_{i_{p}}} \\
\cdot\left(\prod_{\tau=1}^{i_{p}-1} f_{\lambda_{\tau i_{p}}}\left(z_{\tau}, z_{i_{p}}\right)-\prod_{\tau=1}^{i_{p}-1} g_{\lambda_{\tau i_{p}}}\left(z_{\tau}, z_{i_{p}}\right)\right) \\
=\left(\prod_{\tau=i_{p}+1}^{j_{p}-1} g_{\lambda_{\tau j_{p}}}\left(z_{\tau}, z_{i_{p}}\right)\right) \sum_{\tau=1}^{i_{p}-1} \frac{\lambda_{\tau i_{p}}+\left|\lambda_{\tau i_{p}}\right|}{2} \delta\left(z_{\tau} / z_{i_{p}}\right) \delta\left(z_{j_{p}} / z_{i_{p}}\right) .
\end{gathered}
$$


Now we substitute (3.51) into (3.48), and note that (by Lemma 3.15) for $q \neq i_{p}, j_{p}$

$$
\begin{gathered}
\left.f_{-\left(\gamma_{i_{p}}, \gamma_{q}\right)}\left(z_{i_{p}}, z_{q}\right) f_{-\left(\gamma_{j_{p}}, \gamma_{q}\right)}\left(z_{j_{p}}, z_{q}\right)\right|_{z_{j_{p}}=z_{i_{p}}}=1, \\
\left.g_{-\left(\gamma_{i_{p}}, \gamma_{q}\right)}\left(z_{i_{p}}, z_{q}\right) g_{-\left(\gamma_{j_{p}}, \gamma_{q}\right)}\left(z_{j_{p}}, z_{q}\right)\right|_{z_{j_{p}}=z_{i_{p}}}=1, \\
g_{-\left(\gamma_{q}, \gamma_{j_{p}}\right)}\left(z_{q}, z_{i_{p}}\right) f_{-\left(\gamma_{i_{p}}, \gamma_{q}\right)}\left(z_{i_{p}}, z_{q}\right)=(-1)^{\left(\gamma_{i_{p}}, \gamma_{q}\right)}, \\
g_{-\left(\gamma_{q}, \gamma_{j_{p}}\right)}\left(z_{q}, z_{i_{p}}\right) g_{-\left(\gamma_{i_{p}}, \gamma_{q}\right)}\left(z_{i_{p}}, z_{q}\right)=(-1)^{\left(\gamma_{i_{p}}, \gamma_{q}\right)},
\end{gathered}
$$

so we obtain from (3.48)

$$
\begin{aligned}
\bar{Q}\left(z_{1}, \cdots, z_{k}\right) & =\prod_{m=1}^{l}\left((D \delta)\left(z_{j_{m}} / z_{i_{m}}\right)\left\{\prod_{\substack{\tau=1 \\
\tau \neq i_{m}}}^{j_{m}-1} g_{-\left(\gamma_{\tau}, \gamma_{j_{m}}\right)}\left(z_{\tau}, z_{j_{m}}\right)\right) F_{i_{m}}\right\} \prod_{n=1}^{s} F_{t_{n}} \\
& =\left(\prod_{\nu=i_{p}+1}^{j_{p}-1} g_{\lambda_{\nu j_{p}}}\left(z_{\nu}, z_{i_{p}}\right)\right) \sum_{\tau=1}^{i_{p}-1} \frac{\lambda_{\tau i_{p}}+\left|\lambda_{\tau i_{p}}\right|}{2} \delta\left(z_{\tau} / z_{i_{p}}\right) \delta\left(z_{j_{p}} / z_{i_{p}}\right) \\
& \cdot \prod_{\substack{m=1 \\
m \neq p}}^{l}\left\{(D \delta)\left(z_{j_{m}} / z_{i_{m}}\right)\left(\prod_{\substack{\tau=1 \\
\tau \neq i_{m}}}^{j_{m}-1} g_{-\left(\gamma_{\tau}, \gamma_{j_{m}}\right)}\left(z_{\tau}, z_{j_{m}}\right)\right) F_{i_{m}}\right\} \prod_{n=1}^{s} F_{t_{n}} \\
& =\left(\sum_{\tau=1}^{i_{p}-1} \frac{\lambda_{\tau i_{p}}+\left|\lambda_{\tau i_{p}}\right|}{2} \delta\left(z_{\tau} / z_{i_{p}}\right)\right)\left(\prod_{\substack{m=1 \\
m \neq p}}^{l}(D \delta)\left(z_{j_{m}} / z_{i_{m}}\right)\right) \cdot \Lambda,
\end{aligned}
$$

where

$$
\begin{aligned}
\Lambda= & \left(\prod_{\nu=i_{p}+1}^{j_{p}-1} g_{\lambda_{\nu j_{p}}}\left(z_{\nu}, z_{i_{p}}\right)\right) \prod_{\substack{m=1 \\
m \neq p}}^{l}\left(\prod_{\substack{\tau=1 \\
\tau \neq i_{m}}}^{j_{m}-1} g_{\lambda_{\tau j_{m}}}\left(z_{\tau}, z_{j_{m}}\right)\right) \\
& \cdot \prod_{\substack{m=1 \\
m \neq p}}^{l}\left(\prod_{\tau=1}^{i_{m}-1} f_{\lambda_{\tau i m}}\left(z_{\tau}, z_{i_{m}}\right)-\prod_{\tau=1}^{i_{m}-1} g_{\lambda_{\tau i_{m}}}\left(z_{\tau}, z_{i_{m}}\right)\right) \\
& \cdot \prod_{n=1}^{s}\left(\prod_{\tau=1}^{t_{n}-1} f_{\lambda_{\tau t_{n}}}\left(z_{\tau}, z_{t_{n}}\right)-\prod_{\tau=1}^{t_{n}-1} g_{\lambda_{\tau t_{n}}}\left(z_{\tau}, z_{t_{n}}\right)\right) \delta\left(z_{j_{p}} / z_{i_{p}}\right) .
\end{aligned}
$$

Let $\Delta:=\left\{i_{m}, j_{m}, t_{n} \mid 1 \leq m \leq l, 1 \leq n \leq s, m \neq p\right\}$. Recall that $\left(i_{1}, \ldots, i_{l}, j_{1}, \ldots\right.$, $\left.j_{l}, t_{1}, \ldots, t_{s}\right)$ is a permutation of $(1,2, \ldots, k)$, we have $\Delta=\{\mu \mid 1 \leq \mu \leq k, \mu \neq$ $\left.i_{p}, \mu \neq j_{p}\right\}$. Let $\Delta_{0}:=\left\{i_{m}, j_{m}, t_{n} \in \Delta \mid i_{p}<i_{m}, j_{m}, t_{n}<j_{p}\right\}$. Then $\Delta_{0}=$ $\left\{i_{p}+1, \ldots, j_{p}-1\right\}$. Now we rewrite $\Lambda$ in the following form:

$$
\Lambda=\left(\prod_{\nu=i_{p}+1}^{j_{p}-1} g_{\lambda_{\nu j_{p}}}\left(z_{\nu}, z_{i_{p}}\right)\right) \cdot \Lambda_{1} \cdot \Lambda_{2}
$$


where

$$
\begin{array}{r}
\Lambda_{1}=\prod_{\substack{m=1, m \neq p \\
j_{m} \in \Delta_{0}}}^{l}\left(\prod_{\substack{\tau=1 \\
\tau \neq i_{m}}}^{j_{m}-1} g_{\lambda_{\tau j_{m}}}\left(z_{\tau}, z_{j_{m}}\right)\right) \\
\cdot \prod_{\substack{m=1, m \neq p \\
i_{m} \in \Delta_{0}}}^{l}\left(\prod_{\tau=1}^{i_{m}-1} f_{\lambda_{\tau i_{m}}}\left(z_{\tau}, z_{i_{m}}\right)-\prod_{\tau=1}^{i_{m}-1} g_{\lambda_{\tau i_{m}}}\left(z_{\tau}, z_{i_{m}}\right)\right) \\
\cdot \prod_{\substack{n=1 \\
t_{n} \in \Delta_{0}}}^{s}\left(\prod_{\tau=1}^{t_{n}-1} f_{\lambda_{\tau t_{n}}}\left(z_{\tau}, z_{t_{n}}\right)-\prod_{\tau=1}^{t_{n}-1} g_{\lambda_{\tau t_{n}}}\left(z_{\tau}, z_{t_{n}}\right)\right),
\end{array}
$$

and

$$
\begin{array}{r}
\Lambda_{2}=\prod_{\substack{m=1, m \neq p \\
j_{m} \in \Delta \backslash \Delta_{0}}}^{l}\left(\prod_{\substack{\tau=1 \\
\tau \neq i_{m}}}^{l} g_{\lambda_{\tau_{m}}}\left(z_{\tau}, z_{j_{m}}\right)\right) \\
\cdot \prod_{\substack{m=1, m \neq p \\
i_{m} \in \Delta \backslash \Delta_{0}}}^{l}\left(\prod_{\substack{i_{m}-1 \\
j_{m}-1}}^{l} f_{\lambda_{\tau i_{m}}}\left(z_{\tau}, z_{i_{m}}\right)-\prod_{\tau=1}^{i_{m}-1} g_{\lambda_{\tau i_{m}}}\left(z_{\tau}, z_{i_{m}}\right)\right) \\
\cdot \prod_{\substack{n=1 \\
t_{n} \in \Delta \backslash \Delta_{0}}}^{s}\left(\prod_{\tau=1}^{t_{n}-1} f_{\lambda_{\tau t_{n}}}\left(z_{\tau}, z_{t_{n}}\right)-\prod_{\tau=1}^{t_{n}-1} g_{\lambda_{\tau t_{n}}}\left(z_{\tau}, z_{t_{n}}\right)\right) \delta\left(z_{j_{p}} / z_{i_{p}}\right) .
\end{array}
$$

It is clear that

$$
\begin{array}{r}
\Lambda_{2}=\prod_{\substack{m=1, m \neq p \\
j_{m} \in \Delta \backslash \Delta_{0}}}^{l}\left(\prod_{\substack{\tau=1 \\
\tau \neq i_{m}, i_{p}, j_{p}}}^{l} g_{\lambda_{\tau j_{m}}}\left(z_{\tau}, z_{j_{m}}\right)\right) \\
\cdot \prod_{\substack{m=1, m \neq p \\
i_{m} \in \Delta \backslash \Delta_{0}}}^{l}\left(\prod_{\substack{\tau=1 \\
\tau \neq i_{p}, j_{p}}}^{j_{m}-1} f_{\lambda_{\tau i_{m}}}\left(z_{\tau}, z_{i_{m}}\right)-\prod_{\substack{\tau=1 \\
\tau \neq i_{p}, j_{p}}}^{i_{m}-1} g_{\lambda_{\tau i_{m}}}\left(z_{\tau}, z_{i_{m}}\right)\right) \\
\prod_{\substack{n=1 \\
t_{n} \in \Delta \backslash \Delta_{0}}}^{s}\left(\prod_{\substack{\tau=1 \\
\tau \neq i_{p}, j_{p}}}^{i_{n}-1} f_{\lambda_{\tau t_{n}}}\left(z_{\tau}, z_{t_{n}}\right)-\prod_{\substack{\tau=1 \\
\tau \neq i_{p}, j_{p}}}^{t_{n}-1} g_{\lambda_{\tau t_{n}}}\left(z_{\tau}, z_{t_{n}}\right)\right) \delta\left(z_{j_{p}} / z_{i_{p}}\right),
\end{array}
$$


because in the term $\prod_{\substack{m=1, m \neq p \\ j_{m} \in \Delta \backslash \Delta}}^{l} \prod_{\substack{\tau=1 \\ \tau \neq i_{m}}}^{j_{m}-1} g_{\lambda_{\tau j_{m}}}\left(z_{\tau}, z_{j_{m}}\right)$ of $\Lambda_{2}$, if $j_{m}<i_{p}$, then $\tau \neq i_{p}$, $\tau \neq j_{p}$, while if $j_{m}>j_{p}$, then

$$
\begin{gathered}
\prod_{\substack{\tau=1 \\
\tau \neq i_{m}}}^{j_{m}-1} g_{\lambda_{\tau_{m}}}\left(z_{\tau}, z_{j_{m}}\right) \delta\left(z_{j_{p}} / z_{i_{p}}\right) \\
=g_{\lambda_{i_{p} j_{m}}}\left(z_{i_{p}}, z_{j_{m}}\right) g_{\lambda_{j_{p} j_{m}}}\left(z_{j_{p}}, z_{j_{m}}\right)\left(\prod_{\substack{\tau=1 \\
\tau \neq i_{m}, i_{p}, j_{p}}}^{j_{m}-1} g_{\lambda_{\tau_{m}}}\left(z_{\tau}, z_{j_{m}}\right)\right) \delta\left(z_{j_{p}} / z_{i_{p}}\right) \\
=\delta\left(z_{j_{p}} / z_{i_{p}}\right) \prod_{\substack{\tau=1 \\
\tau \neq i_{m}, i_{p}, j_{p}}}^{j_{m}-1} g_{\lambda_{\tau j_{m}}}\left(z_{\tau}, z_{j_{m}}\right)
\end{gathered}
$$

where we have used (3.52), thus in all cases

$$
\begin{gathered}
\delta\left(z_{j_{p}} / z_{i_{p}}\right) \prod_{\substack{m=1, m \neq p \\
j_{m} \in \Delta \backslash \Delta_{0}}}^{l} \prod_{\substack{\tau=1 \\
\tau \neq i_{m}}}^{j_{m}-1} g_{\lambda_{\tau j_{m}}}\left(z_{\tau}, z_{j_{m}}\right) \\
=\delta\left(z_{j_{p}} / z_{i_{p}}\right) \prod_{\substack{m=1, m \neq p \\
j_{m} \in \Delta \backslash \Delta_{0}}}^{l} \prod_{\substack{\tau=1 \\
\tau \neq i_{m}, i_{p}, j_{p}}}^{j_{m}-1} g_{\lambda_{\tau j_{m}}}\left(z_{\tau}, z_{j_{m}}\right),
\end{gathered}
$$

and similarly for other terms in $\Lambda_{2}$ we have

$$
\begin{aligned}
& \prod_{\substack{m=1, m \neq p \\
i_{m} \in \Delta \backslash \Delta_{0}}}^{l}\left(\prod_{\tau=1}^{i_{m}-1} f_{\lambda_{\tau i_{m}}}\left(z_{\tau}, z_{i_{m}}\right)-\prod_{\tau=1}^{i_{m}-1} g_{\lambda_{\tau_{m}}}\left(z_{\tau}, z_{i_{m}}\right)\right) \delta\left(z_{j_{p}} / z_{i_{p}}\right) \\
= & \prod_{\substack{m=1, m \neq p \\
i_{m} \in \Delta \backslash \Delta \Delta_{0}}}^{l}\left(\prod_{\substack{\tau=1 \\
\tau \neq i_{p}, j_{p}}}^{i_{m}-1} f_{\lambda_{\tau i_{m}}}\left(z_{\tau}, z_{i_{m}}\right)-\prod_{\substack{\tau=1 \\
\tau \neq i_{p}, j_{p}}}^{i_{m}-1} g_{\lambda_{\tau i_{m}}}\left(z_{\tau}, z_{i_{m}}\right)\right) \delta\left(z_{j_{p}} / z_{i_{p}}\right),
\end{aligned}
$$

and

$$
\begin{aligned}
& \prod_{\substack{n=1 \\
t_{n} \in \Delta \backslash \Delta_{0}}}^{s}\left(\prod_{\tau=1}^{t_{n}-1} f_{\lambda_{\tau t_{n}}}\left(z_{\tau}, z_{t_{n}}\right)-\prod_{\tau=1}^{t_{n}-1} g_{\lambda_{\tau t_{n}}}\left(z_{\tau}, z_{t_{n}}\right)\right) \delta\left(z_{j_{p}} / z_{i_{p}}\right) \\
= & \prod_{\substack{n=1 \\
t_{n} \in \Delta \backslash \Delta_{0}}}^{s}\left(\prod_{\substack{\tau=1 \\
\tau \neq i_{p}, j_{p}}}^{t_{n}-1} f_{\lambda_{\tau t_{n}}}\left(z_{\tau}, z_{t_{n}}\right)-\prod_{\substack{\tau=1 \\
\tau \neq i_{p}, j_{p}}}^{t_{n}-1} g_{\lambda_{\tau t_{n}}}\left(z_{\tau}, z_{t_{n}}\right)\right) \delta\left(z_{j_{p}} / z_{i_{p}}\right),
\end{aligned}
$$


as required. Next we consider the term $\left(\prod_{\nu=i_{p}+1}^{j_{p}-1} g_{\lambda_{\nu j_{p}}}\left(z_{\nu}, z_{i_{p}}\right)\right) \cdot \Lambda_{1}$ in (3.55). Recall that $\Delta_{0}=\left\{i_{p}+1, \ldots, j_{p}-1\right\}=\left\{i_{m}, j_{m}, t_{n} \in \Delta \mid i_{p}<i_{m}, j_{m}, t_{n}<j_{p}\right\}$, so we have

$$
\begin{gathered}
\left(\prod_{\nu=i_{p}+1}^{j_{p}-1} g_{\lambda_{\nu j_{p}}}\left(z_{\nu}, z_{i_{p}}\right)\right) \cdot \Lambda_{1}=\left(\prod_{\nu \in \Delta_{0}} g_{\lambda_{\nu j_{p}}}\left(z_{\nu}, z_{i_{p}}\right)\right) \cdot \Lambda_{1} \\
=\left(\prod_{j_{m} \in \Delta_{0}} g_{\lambda_{j_{m} j_{p}}}\left(z_{j_{m}}, z_{i_{p}}\right)\right)\left(\prod_{i_{m} \in \Delta_{0}} g_{\lambda_{i_{m} j_{p}}}\left(z_{i_{m}}, z_{i_{p}}\right)\right)\left(\prod_{t_{n} \in \Delta_{0}} g_{\lambda_{t_{n} j_{p}}}\left(z_{t_{n}}, z_{i_{p}}\right)\right) \cdot \Lambda_{1},
\end{gathered}
$$

and this equals

$$
\begin{gathered}
\prod_{\substack{m=1, m \neq p \\
j_{m} \in \Delta_{0}}}^{l}\left(g_{\lambda_{j_{m} j_{p}}}\left(z_{j_{m}}, z_{i_{p}}\right) \prod_{\substack{\tau \neq 1 \\
\tau \neq i_{m}}}^{j_{m}-1} g_{\lambda_{\tau j_{m}}}\left(z_{\tau}, z_{j_{m}}\right)\right) \\
\cdot \prod_{\substack{m=1, m \neq p \\
i_{m} \in \Delta_{0}}}^{l}\left(g_{\lambda_{i_{m} j_{p}}}\left(z_{i_{m}}, z_{i_{p}}\right) \prod_{\tau=1}^{i_{m}-1} f_{\lambda_{\lambda_{m}}}\left(z_{\tau}, z_{i_{m}}\right)\right. \\
\left.-g_{\lambda_{i_{m} j_{p}}}\left(z_{i_{m}}, z_{i_{p}}\right) \prod_{\tau=1}^{i_{m}-1} g_{\lambda_{\tau i_{m}}}\left(z_{\tau}, z_{i_{m}}\right)\right) \\
\prod_{\substack{n=1 \\
t_{n} \in \Delta_{0}}}^{s}\left(g_{\lambda_{t_{n} j_{p}}}\left(z_{t_{n}}, z_{i_{p}}\right) \prod_{\tau=1}^{t_{n}-1} f_{\lambda_{\tau t_{n}}}\left(z_{\tau}, z_{t_{n}}\right)-g_{\lambda_{t_{n} j_{p}}}\left(z_{t_{n}}, z_{i_{p}}\right) \prod_{\tau=1}^{t_{n}-1} g_{\lambda_{\tau t_{n}}}\left(z_{\tau}, z_{t_{n}}\right)\right) .
\end{gathered}
$$

In (3.57) the term

$$
\begin{gathered}
g_{\lambda_{j_{m} j_{p}}}\left(z_{j_{m}}, z_{i_{p}}\right) \prod_{\substack{\tau=1 \\
\tau \neq i_{m}}}^{j_{m}-1} g_{\lambda_{\tau j_{m}}}\left(z_{\tau}, z_{j_{m}}\right) \\
=g_{\lambda_{j_{m} j_{p}}}\left(z_{j_{m}}, z_{i_{p}}\right) g_{\lambda_{i_{p} j_{m}}}\left(z_{i_{p}}, z_{j_{m}}\right) \prod_{\substack{\tau=1 \\
\tau \neq i_{m}, i_{p}, j_{p}}}^{j_{m}-1} g_{\lambda_{\tau j_{m}}}\left(z_{\tau}, z_{j_{m}}\right) \\
=(-1)^{\left(\gamma_{i_{p}}, \gamma_{j_{m}}\right)} \prod_{\substack{\tau=1 \\
\tau \neq i_{m}, i_{p}, j_{p}}}^{j_{\lambda_{m}-1}} g_{\lambda_{\tau j_{m}}}\left(z_{\tau}, z_{j_{m}}\right),
\end{gathered}
$$


where we have used (3.52). Moreover, the second term in (3.57)

$$
\begin{gathered}
g_{\lambda_{i_{m} j_{p}}}\left(z_{i_{m}}, z_{i_{p}}\right) \prod_{\tau=1}^{i_{m}-1} f_{\lambda_{\tau i_{m}}}\left(z_{\tau}, z_{i_{m}}\right)-g_{\lambda_{i_{m} j_{p}}}\left(z_{i_{m}}, z_{i_{p}}\right) \prod_{\tau=1}^{i_{m}-1} g_{\lambda_{\tau i_{m}}}\left(z_{\tau}, z_{i_{m}}\right) \\
=g_{\lambda_{i_{m} j_{p}}}\left(z_{i_{m}}, z_{i_{p}}\right) f_{\lambda_{i_{p} i_{m}}}\left(z_{i_{p}}, z_{i_{m}}\right) \prod_{\substack{\tau=1 \\
\tau \neq i_{p}, j_{p}}}^{i_{m}-1} f_{\lambda_{\tau i_{m}}}\left(z_{\tau}, z_{i_{m}}\right) \\
-g_{\lambda_{i_{m} j_{p}}}\left(z_{i_{m}}, z_{i_{p}}\right) g_{\lambda_{i_{p} i_{m}}}\left(z_{i_{p}}, z_{i_{m}}\right) \prod_{\substack{\tau=1 \\
\tau \neq i_{p}, j_{p}}}^{i_{m}-1} g_{\lambda_{\tau i_{m}}}\left(z_{\tau}, z_{i_{m}}\right) \\
=(-1)^{\left(\gamma_{i_{p}}, \gamma_{i_{m}}\right)}\left(\prod_{\substack{\tau=1 \\
\tau \neq i_{p}, j_{p}}}^{i_{\lambda_{m}-1}} f_{\lambda_{i_{m}}}\left(z_{\tau}, z_{i_{m}}\right)-\prod_{\substack{\tau=1 \\
\tau \neq i_{p}, j_{p}}}^{i_{m}-1} g_{\lambda_{\lambda_{i_{m}}}}\left(z_{\tau}, z_{i_{m}}\right)\right),
\end{gathered}
$$

where we have used (3.52). Similarly, one argues with the last term in (3.57) to obtain

$$
\begin{array}{r}
g_{\lambda_{t_{n} j_{p}}}\left(z_{t_{n}}, z_{i_{p}}\right) \prod_{\tau=1}^{t_{n}-1} f_{\lambda_{\tau t_{n}}}\left(z_{\tau}, z_{t_{n}}\right)-g_{\lambda_{t_{n} j_{p}}}\left(z_{t_{n}}, z_{i_{p}}\right) \prod_{\tau=1}^{t_{n}-1} g_{\lambda_{\tau t_{n}}}\left(z_{\tau}, z_{t_{n}}\right) \\
=(-1)^{\left(\gamma_{i_{p}}, \gamma_{t_{n}}\right)}\left(\prod_{\substack{\tau=1 \\
\tau \neq \bar{p}_{p}, j_{p}}}^{t_{n}-1} f_{\lambda_{\tau t_{n}}}\left(z_{\tau}, z_{t_{n}}\right)-\prod_{\substack{\tau=1 \\
\tau \neq i_{p}, j_{p}}}^{t_{n}-1} g_{\lambda_{\tau t_{n}}}\left(z_{\tau}, z_{t_{n}}\right)\right) .
\end{array}
$$

Therefore, by the above three identities we obtain from (3.57)

$$
\begin{gathered}
\left(\prod_{\nu=i_{p}+1}^{j_{p}-1} g_{\lambda_{\nu j_{p}}}\left(z_{\nu}, z_{i_{p}}\right)\right) \cdot \Lambda_{1}=\prod_{\substack{m=1, m \neq p \\
j_{m} \in \Delta_{0}}}^{l}\left((-1)^{\left(\gamma_{i_{p}}, \gamma_{j_{m}}\right)} \prod_{\substack{\tau=1 \\
\tau \neq i_{m}, i_{p}, j_{p}}}^{j_{m}-1} g_{\lambda_{\tau j_{m}}}\left(z_{\tau}, z_{j_{m}}\right)\right) \\
\cdot \prod_{\substack{m=1, m \neq p \\
i_{m} \in \Delta_{0}}}^{l}\left((-1)^{\left(\gamma_{i_{p}}, \gamma_{i_{m}}\right)} \prod_{\substack{\tau=1 \\
\tau \neq i_{p}, j_{p}}}^{i_{m}-1} f_{\lambda_{\tau i_{m}}}\left(z_{\tau}, z_{i_{m}}\right)\right. \\
\cdot \prod_{\substack{n=1 \\
t_{n} \in \Delta_{0}}}^{s}\left((-1)^{\left(\gamma_{i_{p}}, \gamma_{i_{m}}\right)} \prod_{\substack{\tau=1 \\
\tau \neq i_{p}, j_{p}}}^{i_{m}-1} g_{\lambda_{\tau i}}\left(z_{\tau}, z_{i_{m}}\right)\right) \\
\left(\gamma^{\left(\gamma_{i_{p}}, \gamma_{t_{n}}\right)} \prod_{\substack{\tau=1 \\
\tau \neq i_{p}, j_{p}}}^{t_{n}-1} f_{\lambda_{\tau t_{n}}}\left(z_{\tau}, z_{t_{n}}\right)-(-1)^{\left(\gamma_{i_{p}}, \gamma_{t_{n}}\right)} \prod_{\substack{\tau=1 \\
\tau \neq i_{p}, j_{p}}}^{t_{n}-1} g_{\lambda_{\tau t_{n}}}\left(z_{\tau}, z_{t_{n}}\right)\right)
\end{gathered}
$$


and this equals

$$
\begin{aligned}
& (-1)^{\left(\gamma_{i_{p}}, \sum_{i_{p}<\nu<j_{p}} \gamma_{\nu}\right)} \prod_{\substack{m=1, m \neq p \\
j_{m} \in \Delta_{0}}}^{l}\left(\prod_{\substack{\tau=1 \\
\tau \neq i_{m}, i_{p}, j_{p}}}^{j_{m}-1} g_{\lambda_{\tau j_{m}}}\left(z_{\tau}, z_{j_{m}}\right)\right) \\
& \cdot \prod_{\substack{m=1, m \neq p \\
i_{m} \in \Delta_{0}}}^{l}\left(\prod_{\substack{\tau=1 \\
\tau \neq i_{p}, j_{p}}}^{i_{m}-1} f_{\lambda_{\tau i_{m}}}\left(z_{\tau}, z_{i_{m}}\right)-\prod_{\substack{\tau=1 \\
\tau \neq i_{p}, j_{p}}}^{i_{m}-1} g_{\lambda_{\tau i_{m}}}\left(z_{\tau}, z_{i_{m}}\right)\right) \\
& \cdot \prod_{\substack{n=1 \\
t_{n} \in \Delta_{0}}}^{s}\left(\prod_{\substack{\tau=1 \\
\tau \neq i_{p}, j_{p}}}^{t_{n}-1} f_{\lambda_{\tau t_{n}}}\left(z_{\tau}, z_{t_{n}}\right)-\prod_{\substack{\tau=1 \\
\tau \neq i_{p}, j_{p}}}^{t_{n}-1} g_{\lambda_{\tau t_{n}}}\left(z_{\tau}, z_{t_{n}}\right)\right) .
\end{aligned}
$$

We combine (3.56) and (3.58) to obtain

$$
\begin{aligned}
& \Lambda=\left(\prod_{\nu=i_{p}+1}^{j_{p}-1} g_{\lambda_{\nu j_{p}}}\left(z_{\nu}, z_{i_{p}}\right)\right) \cdot \Lambda_{1} \cdot \Lambda_{2} \\
& =(-1)^{\left(\gamma_{i_{p}}, \sum_{i_{p}<\nu<j_{p}} \gamma_{\nu}\right)} \prod_{m=1, m \neq p}^{l}\left(\prod_{\substack{\tau=1 \\
\tau \neq i_{m}, i_{p}, j_{p}}}^{l} g_{\lambda_{\tau j_{m}}}\left(z_{\tau}, z_{j_{m}}\right)\right) \\
& \cdot \prod_{m=1, m \neq p}^{l}\left(\prod_{\substack{\tau=1 \\
\tau \neq i_{p}, j_{p}}}^{i_{m}-1} f_{\lambda_{\tau i_{m}}}\left(z_{\tau}, z_{i_{m}}\right)-\prod_{\substack{\tau=1 \\
\tau \neq i_{p}, j_{p}}}^{i_{m}-1} g_{\lambda_{\tau i}}\left(z_{\tau}, z_{i_{m}}\right)\right) \\
& \cdot \prod_{n=1}^{s}\left(\prod_{\substack{\tau=1 \\
\tau \neq i_{p}, j_{p}}}^{t_{n}-1} f_{\lambda_{\tau t_{n}}}\left(z_{\tau}, z_{t_{n}}\right)-\prod_{\substack{\tau=1 \\
\tau \neq i_{p}, j_{p}}}^{t_{n}-1} g_{\lambda_{\tau t_{n}}}\left(z_{\tau}, z_{t_{n}}\right)\right) .
\end{aligned}
$$

Finally, we substitute (3.59) into (3.53) to obtain

$$
\begin{aligned}
& \bar{Q}\left(z_{1}, \cdots, z_{k}\right)=(-1)^{\left(\gamma_{i_{p}}, \sum_{i_{p}<q<j_{p}} \gamma_{q}\right)} \sum_{\tau=1}^{i_{p}-1} \frac{\lambda_{\tau i_{p}}+\left|\lambda_{\tau i_{p}}\right|}{2} \delta\left(z_{\tau} / z_{i_{p}}\right) \delta\left(z_{j_{p}} / z_{i_{p}}\right) \\
& \cdot \prod_{\substack{m=1 \\
m \neq p}}^{l}\left\{( D \delta ) ( z _ { j _ { m } } / z _ { i _ { m } } ) ( \prod _ { \substack { \tau = 1 \\
\tau \neq i _ { m } , i _ { p } , j _ { p } } } ^ { j _ { m } - 1 } g _ { - ( \gamma _ { \tau } , \gamma _ { j _ { m } } ) } ( z _ { \tau } , z _ { j _ { m } } ) ) \left(\prod_{\substack{\tau=1 \\
\tau \neq i_{p}, j_{p}}}^{i_{m}-1} f_{-\left(\gamma_{\tau}, \gamma_{i_{m}}\right)}\left(z_{\tau}, z_{i_{m}}\right)\right.\right. \\
& \left.\left.\cdot \prod_{n=1}^{i_{m}-1} g_{-\left(\gamma_{\tau}, \gamma_{i_{m}}\right)}\left(z_{\tau}, z_{i_{m}}\right)\right)\right\} \\
& =\left(\prod_{\substack{\tau=1 \\
\tau \neq i_{p}, j_{p}}}^{s} f_{-\left(\gamma_{\tau}, \gamma_{t_{n}}\right)}\left(z_{\tau}, z_{t_{n}}\right)-\prod_{\substack{\tau=1 \\
\tau \neq i_{p}, j_{p}}}^{t_{n}-1} g_{-\left(\gamma_{\tau}, \gamma_{t_{n}}\right)}\left(z_{\tau}, z_{t_{n}}\right)\right) \\
& \cdot \bar{Q}\left(z_{1}, \ldots, \hat{z}_{i_{p}}, \ldots, \hat{z}_{j_{p}}, \ldots, z_{k}\right),
\end{aligned}
$$


where ' $\leadsto$ ' means the term is deleted. But $\bar{Q}\left(z_{1}, \ldots, \hat{z}_{i_{p}}, \ldots, \hat{z}_{j_{p}}, \ldots, z_{k}\right)$ is zero by induction. This completes the proof of subcase B and also the theorem.

Therefore, we have the following result which generalizes the statement of Corollary 3.46, and is our generalization of the square length two cut off theorem of Goddard and Olive.

Corollary 3.60. Let $k \geq 2, \beta_{1}, \ldots, \beta_{k} \in\left\{\alpha_{j}^{+}, \alpha_{j}^{-}, \not \mid j \in J\right\}, \gamma_{1}, \ldots, \gamma_{k} \in \Gamma$.We assume that $\gamma_{i}=\alpha_{j}$ if $\beta_{i}=\alpha_{j}^{+}$for some $j \in J$, or $\gamma_{i}=-\alpha_{j}$ if $\beta_{i}=\alpha_{j}^{-}$for some $j \in J$, where $1 \leq i \leq k$. Then $\left[X^{\left(\beta_{k}\right)}\left(\gamma_{k}, z_{k}\right), \cdots, X^{\left(\beta_{1}\right)}\left(\gamma_{1}, z_{1}\right)\right]=0$, or equivalently,

$$
\left[x_{n_{k}}^{\left(\beta_{k}\right)}\left(\gamma_{k}\right), \cdots, x_{n_{1}}^{\left(\beta_{1}\right)}\left(\gamma_{1}\right)\right]=0
$$

for all $n_{1}, \ldots, n_{k} \in \mathbf{Z}$, provided that $\left(\gamma_{i}, \gamma_{i}\right) \leq 2$, for $i=1, \ldots, k$, and $\left(\sum_{i=1}^{k} \gamma_{i}\right.$, $\left.\sum_{i=1}^{k} \gamma_{i}\right)>2$.

Proof. This follows immediately from Proposition 3.32, Corollary 3.43 and Theorem 3.47.

Recall that the Lie algebra $\mathcal{G}(A)$ attached to the matrix $A=\left(a_{i j}\right)_{i, j \in I}$ is generated by the elements $\left\{e_{i}, f_{i}, h_{i}, \quad i \in I\right\}$, with the relations (R1) through (R4). Also, recall that $\mathcal{L}(A)=\mathcal{G}(A) / \mathcal{R}(A)$. We now form the vertex operators $X^{(\phi)}\left(\alpha_{i}, z\right), X^{(\phi)}\left(-\alpha_{i}, z\right)$ for $i \in I \backslash J$, and $X^{\left(\alpha_{j}^{+}\right)}\left(\alpha_{j}, z\right), X^{\left(\alpha_{j}^{-}\right)}\left(-\alpha_{j}, z\right)$ for $j \in J$. With any choice $\vec{n}=\left(n_{i}\right)_{i \in I} \in \mathbf{Z}^{I}$, we form the Lie algebra $\mathcal{G}(\vec{n}, \Gamma)$ which is generated by the operators $\left\{x_{n_{i}}^{(\phi)}\left(\alpha_{i}\right), x_{-n_{i}}^{(\phi)}\left(-\alpha_{i}\right)\right\}_{i \in I \backslash J}$ and $\left\{x_{n_{j}}^{\left(\alpha_{j}^{+}\right)}\left(\alpha_{j}\right), x_{-n_{j}}^{\left(\alpha_{j}^{-}\right)}\left(-\alpha_{j}\right)\right\}_{i \in J}$. We define

$$
\begin{gathered}
\tilde{e}_{i}= \begin{cases}x_{n_{i}}^{(\phi)}\left(\alpha_{i}\right), & \text { if } i \in I \backslash J, \\
x_{n_{i}}^{\left(\alpha_{i}^{+}\right)}\left(\alpha_{i}\right), & \text { if } i \in J,\end{cases} \\
\tilde{f}_{i}= \begin{cases}(-1)^{\frac{1}{2}\left(\alpha_{i}, \alpha_{i}\right)} x_{-n_{i}}^{(\phi)}\left(-\alpha_{i}\right), & \text { if } i \in I \backslash J, \\
(-1)^{\frac{1}{2}\left(\alpha_{i}, \alpha_{i}\right)} x_{-n_{i}}^{\left(\alpha_{-}^{-}\right)}\left(-\alpha_{i}\right), & \text { if } i \in J,\end{cases}
\end{gathered}
$$

and $\tilde{h}_{i}=\left[\tilde{e}_{i}, \tilde{f}_{i}\right]$. Then we have

Lemma 3.63. $\tilde{h}_{i}=n_{i} \phi+\alpha_{i}(0)$, for $i \in I$.

Proof. Since $X^{(\phi)}(\alpha, z)=X(\alpha, z)$ for $\alpha \in \Gamma$, we obtain $\tilde{h}_{i}=n_{i} \phi+\alpha_{i}(0)$ for $i \in I \backslash J$ by Proposition 3.3, while if $i \in J$, the result follows from Corollary 3.39.

Theorem 3.64. Let $A=\left(a_{i j}\right)_{i, j \in I}$ be an admissible matrix satisfying (C1) and (C2), and let $\Gamma$ be the lattice defined from $A$ as in the text. Then the vector space $M(\Gamma)$ affords a representation of the Lie algebra $\mathcal{L}(A)$.

Proof. With the choice $\tilde{e}_{i}, \tilde{f}_{i}, \tilde{h}_{i}, \quad i \in I$ defined by (3.62), Lemma 3.63 and (3.1) imply the relations in (R1). Relations (R2)-(R4) follow from Proposition 3.3 and 
Corollary 3.45. Thus we have a homomorphism $\tilde{\phi}: \quad \mathcal{G}(A) \rightarrow \mathcal{G}(\vec{n}, \Gamma)$ defined by

$$
e_{i} \rightarrow \begin{cases}x_{n_{i}}^{(\phi)}\left(\alpha_{i}\right), & \text { if } i \in I \backslash J \\ x_{n_{i}}^{\left(\alpha_{i}^{+}\right)}\left(\alpha_{i}\right), & \text { if } i \in J\end{cases}
$$

and

$$
f_{i} \rightarrow \begin{cases}(-1)^{\frac{1}{2}\left(\alpha_{i}, \alpha_{i}\right)} x_{-n_{i}}^{(\phi)}\left(-\alpha_{i}\right), & \text { if } i \in I \backslash J \\ (-1)^{\frac{1}{2}\left(\alpha_{i}, \alpha_{i}\right)} x_{-n_{i}}^{\left(\alpha_{\overline{-}}^{-}\right)}\left(-\alpha_{i}\right), & \text { if } i \in J\end{cases}
$$

Therefore, $\vec{n}$ determines a vertex representation of $\mathcal{G}(A)$. Moreover, Corollary 3.60 shows that $\tilde{\phi}\left(\mathcal{G}^{\alpha}(A)\right)=0$ for $\alpha \in \Gamma$ satisfying $(\alpha, \alpha)>2$. Therefore, $\tilde{\phi}$ induces a homomorphism

$$
\phi: \mathcal{L}(A)=\mathcal{G}(A) / \mathcal{R}(A) \rightarrow \mathcal{G}(\vec{n}, \Gamma)
$$

That is, $\vec{n}$ determines a representation of $\mathcal{L}(A)$.

\section{REFERENCES}

[Be] S. Berman, On generators and relations for certain involutory subalgebras of Kac-Moody Lie algebras, Comm. in Algebra. 17(1989), 3165-3185. [MR 91a:17030]

$[\mathrm{BM}] \quad$ S. Berman and R.V. Moody, Lie algebras graded by finite root systems and the intersection matrix algebras of Slodowy, Invent. Math. 108(1992), 323-347. MR 93e:17031

[Bo1] R. Borcherds, Vertex algebras, Kac-Moody algebras, and the monster, Proc. Natl. Acad. Sci. 83(1986), 3068-3071. MR 87m:17033

[Bo2] R. Borcherds, Generalized Kac-Moody Lie algebras, J. Algebra, 115(1988), 501-512. MR 89g:17004

[Bo3] R. Borcherds, Central extensions of the generalized Kac-Moody Lie algebras, J. Algebra, 140(1991), 330-335. MR 92g:17031

[BZ] G. Benkart and E. Zelmanov, Lie algebras graded by finite root systems and intersection matrix algebras, Invent. Math. 126(1996), 1-45. MR 97k:17044

[EMY] S. Eswara Rao, R.V. Moody and T. Yokonuma, Lie algebras and Weyl groups arising from vertex operator representations, Nova J. of Algebra and Geometry, 1(1992), 15-57. MR 93h:17040

[FLM] I.B. Frenkel, J. Lepowsky and A. Meurman, Vertex operator algebras and the Monster, Academic Press, Boston, 1988. MR 90h:17026

[Fr] I.B. Frenkel, Representations of Kac-Moody algebras and dual resonance modules, Lectures in Applied Math. 21(1985), 325-353. MR 87b:17010

[GO] P. Goddard and D. Olive, Algebras, lattices and strings, Vertex operators in mathematics and physics, Publ. Math. Sci. Res. Inst. 3(1985), 51-96, Springer-Verlag. MR 87c:17025

[J1] E. Jurisich, An exposition of the generalized Kac-Moody algebras, Contemporary Math. 194(1996), 121-159. MR 97e:17035

[J2] E. Jurisich, Generalized Kac-Moody Lie algebras, free Lie algebras and the structure of the Monster Lie algebra, J. Pure Appl. Algebra 126 (1998), 233-266. MR 99b:17032

[JLW] E. Jurisich, J. Lepowsky and R.L. Wilson, Realizations of the monster Lie algebra, Selecta Mathematica, new series, 1(1995), 129-161. MR 96e:17059

[K] V.G. Kac, Infinite dimensional Lie algebras, 3rd edition, Cambridge University Press, 1990. MR 92k:17038

[MP] R.V. Moody and A. Pianzola, Lie algebras with triangular decomposition, John Wiley, 1995, New York. MR 96d:17025 
[S11] P. Slodowy, Beyond Kac-Moody algebras and inside, Can. Math. Soc. Conf. Proc. 5(1986), 361-371. CMP 18:10

[S12] P. Slodowy, Kac-Moody algebras, assoziiert Gruppen und Verallgemeinerugen, Habiliation-sschrift, Universitat Bonn, 1984.

Department of Mathematics and Statistics, University of Saskatchewan, Saskatoon, SASkatcheWAN, S7N 5E6 CANAdA

E-mail address: berman@snoopy.usask.ca

Department of Mathematics, University of California, Santa Cruz, California 95064 Current address: Department of Mathematics, College of Charleston, Charleston, South Carolina 29424

E-mail address: jurisiche@cofc.edu

Department of Mathematics, Xiamen University, Xiamen, 361005 Fujian, People's RePUBLIC OF CHINA

E-mail address: tans@jingxian.xmu.edu.cn 